\title{
Beyond Gene Ontology (GO): using biocuration approach to improve the gene nomenclature and functional annotation of rice S-domain kinase subfamily
}

\author{
Sushma Naithani ${ }^{\text {Corresp., } 1}{ }^{1}$, Daemon Dikeman ${ }^{1}$, Priyanka Garg ${ }^{1}$, Noor Al-Bader ${ }^{1}$, Pankaj Jaiswal ${ }^{1}$ \\ ${ }^{1}$ Botany and Plant Pathology, Oregon State University, Corvallis, Oregon, United States of America \\ Corresponding Author: Sushma Naithani \\ Email address: naithans@science.oregonstate.edu
}

The S-domain subfamily of receptor-like kinases (SDRLKs) in plants is poorly characterized. Most members of this subfamily are currently assigned gene function based on the S-locus Receptor Kinase (SRK) from Brassica that acts as the female determinant of selfincompatibility (SI). However, Brassica like SI mechanisms does not exist in most plants. Thus, automated Gene Ontology pipelines are not sufficient for functional annotation of SDRLK subfamily members and lead to erroneous association with the GO biological process of SI. Here, we show that manual bio-curation can help to correct and improve the gene annotations and association with relevant biological processes. Using publicly available genomic and transcriptome datasets, we conducted a detailed analysis of the expansion of the rice (Oryza sativa) SDRLK subfamily, the structure of individual genes and proteins, and their expression.The 144-member SDRLK family in rice consists of 82 receptor-like kinases (67 full-length, 15 truncated), 12 receptor-like proteins, 14 SD kinases, 26 kinase-like, and 10 GnK2 domain-containing kinases and RLKs. Except for nine genes, all other SDRLK family members are transcribed in rice, but they vary in their tissue-specific and stress-response expression profiles. Furthermore, 98 genes show differential expression under biotic stress, and 98 genes show differential expression under abiotic stress conditions, but share 81 genes in common.Our analysis led to the identification of candidate genes likely to play important roles in plant development, pathogen resistance, and abiotic stress tolerance. We propose a nomenclature for 144 SDRLK gene family members based on gene/protein conserved structural features, gene expression profiles, and literature review. Our biocuration approach, rooted in the principles of findability, accessibility, interoperability, and reusability (FAIR), sets forth an example of how manual annotation of large-gene families can fill in the knowledge gap that exists due to the implementation of automated gene ontology (GO) projections, thereby helping to improve the quality and contents of public databases. 


\section{Beyond Gene Ontology (GO): using biocuration approach}

2 to improve the gene nomenclature and functional annotation of rice $\mathrm{S}$-domain 3 kinase subfamily

4

5 Sushma Naithani*, Daemon A. Dikeman, Priyanka Garg, Noor Al-Bader, and Pankaj Jaiswal 6

7 Department of Botany and Plant Pathology, Oregon State University, Corvallis, OR, 97331, 8 USA

9

Corresponding Author: Sushma Naithani Oregon State University, Corvallis, OR-97331, USA Email address: naithans@science.oregonstate.edu 


\section{Abstract}

19 The S-domain subfamily of receptor-like kinases (SDRLKs) in plants is poorly characterized.

20 Most members of this subfamily are currently assigned gene function based on the $S$-locus

21 Receptor Kinase (SRK) from Brassica that acts as the female determinant of self-incompatibility (SI). However, Brassica like SI mechanisms does not exist in most plants. Thus, automated Gene Ontology pipelines are not sufficient for functional annotation of SDRLK subfamily members and lead to erroneous association with the GO biological process of SI. Here, we show that manual bio-curation can help to correct and improve the gene annotations and association with relevant biological processes. Using publicly available genomic and transcriptome datasets, we conducted a detailed analysis of the expansion of the rice (Oryza sativa) SDRLK subfamily, the structure of individual genes and proteins, and their expression. The 144-member SDRLK family in rice consists of 82 receptor-like kinases (67 fulllength, 15 truncated), 12 receptor-like proteins, 14 SD kinases, 26 kinase-like, and $10 \mathrm{GnK} 2$ domain-containing kinases and RLKs. Except for nine genes, all other SDRLK family members are transcribed in rice, but they vary in their tissue-specific and stress-response expression profiles. Furthermore, 98 genes show differential expression under biotic stress, and 98 genes show differential expression under abiotic stress conditions, but share 81 genes in common. Our analysis led to the identification of candidate genes likely to play important roles in plant development, pathogen resistance, and abiotic stress tolerance. We propose a nomenclature for 144 SDRLK gene family members based on gene/protein conserved structural features, gene expression profiles, and literature review. Our biocuration approach, rooted in the principles of findability, accessibility, interoperability, and reusability (FAIR), sets forth an example of how manual annotation of large-gene families can fill in the knowledge gap that exists due to the implementation of automated gene ontology (GO) projections, thereby helping to improve the quality and contents of public databases. 


\section{Introduction}

45 Receptor-like kinases (RLKs) are major players in perceiving and transducing extracellular signals into appropriate cellular responses and have been associated with nearly every aspect of plant growth and development (Becraft et al. 1996; Berckmans et al. 2020; Cartwright et al. 2009; Clark et al. 1997), organ differentiation (Hord et al. 2006; Jinn et al. 2000; Pu et al. 2017; Shpak et al. 2003), plant reproduction (Ahmadi et al. 2016; Escobar-Restrepo et al. 2007; Kachroo et al. 2001; Stein et al. 1991; Yu et al. 2016), and plant's response to biotic (Chen et al. 2006; Fan et al. 2018; Gomez-Gomez \& Boller 2000; Pruitt et al. 2015), and abiotic stresses (Chen et al. 2013; Grison et al. 2019; Ouyang et al. 2010; Pan et al. 2020). The RLKs are encoded by one of the largest gene family, the RLK gene family (e.g., $~ 600$ members in Arabidopsis thaliana and 1,131 in Oryza sativa) (Lehti-Shiu \& Shiu 2012; Shiu et al. 2004).

Typically, an RLK protein consists of an extracellular N-terminal domain followed by a transmembrane domain, and a conserved C-terminal kinase domain. The extracellular domain of RLKs are involved in sensing the extracellular signals via interacting with ligands (i.e., small molecules such as hormones, glycoproteins, or short peptides), and the cytoplasmic Serine/Threonine (Ser/Thr) kinase domain transfers the signal by phosphorylating downstream targets to activate/inactivate the relevant physiological pathways (Wolf 2017). RLKs display great variety in their extracellular domains, consistent with their role in recognizing the diverse ligands, whereas their kinase domains show high conservation in sequence and structure. Thus, based on the structure of their extracellular domains and the phylogenetic relationships between kinase domains, RLKs are grouped into subfamilies, such as leucine-rich repeats (LRRs), Sdomains (SD), Wall-associated kinases (WAKs), etc. (Shiu \& Bleecker 2001a; Shiu \& Bleecker 2001b).

The SDRLKs, the second-largest subfamily of RLKs, share similarity with the extracellular domain (or S-domain) of the $S$-locus receptor kinase (SRK) protein (Shiu \& Bleecker 2001a; Shiu \& Bleecker 2003). SRK was first identified in Brassica stigma epidermis (Stein et al. 1991), where it acts as the female determinant of self-incompatibility (SI) during pollen-pistil interaction in an allele-specific manner (Kachroo et al. 2001). The ligand for SRK is a small peptide, known as S-locus cysteine-rich protein (SCR/SP11), located on the pollen surface (Naithani et al. 2013). A polymorphic $S$-locus region in the Brassica genome encodes both the SRK and SCR (Kachroo et al. 2001; Naithani et al. 2013; Takayama \& Isogai 2003). Upon pollination, SRK interacts with SCR, and a productive interaction leads to activation of the downstream signaling pathway that inhibits pollen germination. Thus, in the Brassicaceae family, selfing is prevented by SI and regulated by the $S$-locus haplotypes. Besides ligand binding (Boggs et al. 2009), the S-domain of SRK is also involved in receptor dimerization (Naithani et al. 2007) and pistil development (Tantikanjana et al. 2009).

Typically, after genome sequencing, based on similarity with SRK, the automated Gene Ontology (GO) pipelines assign electronic annotation to most SDRLK subfamily members as 'SRK' or 'S-domain receptor-like kinase' or 'Similar to SRK protein', and associate with the biological process of 'SI' or 'recognition of pollen' irrespective of their relevance in the given plant species. The partial genes of this family are assigned unknown functions. However, the sporophytic self-incompatibility mechanism does not exist in rice and many other plant species. Recent studies of a few additional genes suggest that this subfamily members act as key 
90 regulators in diverse biological processes. For example, in rice Stress-Induced Kinase 2

91 (OsSIK2) (Chen et al. 2013), OsESG1 (Pan et al. 2020), Large Spike Kinase 1 (OsLSK1) (Zou et

92 al. 2015), Plant Stature Related Kinase 2 (PSRK2) (Li et al. 2018) genes have been shown to

93 play crucial roles in plant development. Arabidopsis CALMODULIN-BINDING RECEPTOR-

94 LIKE PROTEIN KINASE1 (CBRLK1) (Kim et al. 2009), rice PID2 (Chen et al. 2006; Wang et al. 95 2015), and SPL11 Death Suppressor 2 (SDS2) contribute to pathogen resistance and immunity 96 (Fan et al. 2018). Also, rice genes OsSIK2 (Chen et al. 2013), OsESG1 (Pan et al. 2020) have 97 been shown to confer abiotic stress tolerance. Therefore, annotations of SDRLK subfamily genes 98 in the genomic databases are erroneous and is liable to mislead researchers conducting high99 throughput genomic analyses. Often, the SDRLK family genes are ignored as candidate genes 100 for further experimental testing.

101

102

103

104

Accordingly, we propose improved gene nomenclature and descriptions for 144 members of rice (O. sativa) SDRLK subfamily genes using biocuration approach that involves collection of information about structural features, transcription profiles, and subcellular localization of genes and their products from literature and based-on the analysis of publicly available genomics and transcriptome datasets. The improved gene annotation of rice SDRLK subfamily will help rice researchers conducting large-scale genomic analyses in identifying candidate genes involved in in plant developmental processes and stress-response. We are the secondary users of the publicly available and/or previously published rice datasets. However, we are re-using these datasets to ask new questions to define structure and expression profile of rice SDRLK subfamily. Mostly, the primary generators of big genomic data do not fully utilize their data beyond their limited focus on a pathway or biological processes. Therefore, genomic datasets available under findable, accessible, interoperable, and reusable (FAIR) data agreements are a gold mine for other researchers. A growing amount of data mining and secondary analyses of public datasets is recognized by the Pacific Symposium on Biocomputing, which celebrates the impactful metaanalyses through their annual Research Parasite Awards (https://researchparasite.com). Considering the prevalence of large gene families in plant genomes, our approach of re-use and re-analysis of publicly available genomic datasets and manual biocuration could be implemented for improving the annotation of other gene families. \\ 121 Methods}

\section{Updates on rice $S D R L K$ subfamily}

123 Previously, 170 SDRLK subfamily members had been identified in rice (Oryza sativa ssp. japonica cv. Nipponbare) genome (Lehti-Shiu et al. 2009; Shiu et al. 2004) based on the gene models provided by the Michigan State University Rice Genome Annotation Project (MSU) project (http://rice.plantbiology.msu.edu) (27). Over the past ten years, the reference rice gene models have been extensively revised, and the rice research community has adopted improved gene models and gene nomenclature from the Rice Annotation Project (RAP) (https://rapdb.dna.affrc.go.jp) (28). Thus, we revisited models for all 170 genes of the SDRLK family in the MSU dataset, and after removing obsolete, and short genes (encoding peptides of models, we proceeded with 144 genes for further analysis (see Supplementary Table 1). 


\section{Gene structure and protein domain analysis}

135 We used the rice genome browser provided by the Gramene (Tello-Ruiz et al. 2018) to

136 investigate gene and transcript models of 144 SDRLK family members. For genes associated

137 with alternatively spliced transcript isoforms, only the longest canonical transcript was

138 considered. The information about protein domains and GO annotations was retrieved from the

139 protein knowledgebase UniProt (https://www.uniprot.org). The information about the subcellular

140 location of proteins was collected from the Compendium of Crop Proteins with Annotated

141 Locations (http://crop-pal.org) and/or UniProt. If available, the gene/protein structure and/or

142 localization information was further updated based on published studies (e.g., Os06g0494100,

143 Os01g0890100) (Chen et al. 2006; Li et al. 2018). Supplementary Table 1 provides information

144 on the 144 rice SDRLK family (i.e., gene structures, protein domains, subcellular locations, and

145 associated GO biological processes).

146

\section{Tandem gene duplication analysis}

148 We extracted sequences of 144 rice SDRLK family proteins from the Gramene

149 (http://gramene.org). We then built a sequence similarity tree to identify subgroups of genes that

150 are most similar using Clustal Omega (https://www.ebi.ac.uk/Tools/msa/clustalo) (Sievers et al.

151 2011) software (set to default parameters). The tree file saved from Clustal Omega was exported

152 to the Interactive Tree of Life (iToL; https://itol.embl.de) (Letunic \& Bork 2016) website to

153 visualize and add data layers. We further investigated if genes that show high sequence similarity

154 are in the same genomic neighborhood. We uploaded Gene IDs on the $O$. sativa genome browser

155 available at Gramene and then manually inspected the genomic location and flanking regions of

156 individual genes. We defined tandem duplicates based on sequence similarity and genomic

157 location ( $<$ five genes in between).

158

\section{Gene expression analysis and visualization}

160 The publicly available baseline and differential gene expression data of the rice SDRLK family genes used in this study and relevant information e.g. tissue sample, growth and developmental stage of plants, stress treatment and source is provided in Supplementary Table 2. All datasets used in this study contain high quality sequence data with minimum three replicates.

The baseline tissue-specific gene expression data for rice SDRLK genes was extracted from EGEOD-50777 (Anderson et al. 2013), E-MTAB-4123 (Zhang et al. 2014), E-MTAB-2037 (Sakai et al. 2011), and E-MTAB-2039 (Davidson et al. 2012) datasets available from EMBL-EBI Expression Atlas (www.ebi.ac.uk/gxa/plant/experiments). All four datasets are from O. sativa ssp. japonica rice plants grown under normal growth conditions, and together represent expression data for SDRLK family genes from various vegetative and reproductive tissues. The Integrated RNA-Seq Analysis Pipeline (iRAP) developed by EBI Gene Expression Atlas was used for the data analysis including FASTQ QC, alignment, mapping QC and gene expression quantification. Each experiment is manually curated to a high standard and mapped

174 to the most recent assembly of the rice reference genome. The tools employed for genome 
175 alignment, quantification and differential expression include Tophat2/Star, HTSeq-count

176 (Papatheodorou et al. 2020). The normalized gene expression values are for all samples in each

177 dataset are in Transcripts Per Million (TPM) (see Supplementary Table 3). The data from all four

178 files were plotted side-by-side while individual datasets were maintained as an independent

179 block (samples were not clustered based on tissue types). Considering that each tissue or cell-

180 type differs significantly from each other in their mRNA population, we did not make direct

181 quantitative comparisons between the samples and across the datasets. But, made a

182 comparison of expression profile among the SDRLK genes within each tissue sample to

183 summarize baseline tissue-specific expression.

184

185 We found five differential gene expression (log2-fold change 1.0 and adjusted $p \leq=0.05$ )

186 datasets for biotic stress treatments. E-GEOD-61833 (Girija et al. 2017); E-GEOD-36272; E-

187 MTAB-5025 (Zhao et al. 2016); E-MTAB-4406 (Magbanua et al. 2014) datasets are available at

188 the EMBL-EBI Expression Atlas and have been analyzed by the iRAP pipeline similar to

189 baseline tissue-specific data described above, and then subjected to DESeq2 for identifying

190 differentially expressed genes. TheE-MTAB-6402 (ERS2106879-ERS2106902) was previously

191 generated in our laboratory using Illumina HiSeq 3000 (Illumina Inc., USA). The processing of

192 raw sequence data, read alignment to the rice japonica reference cv. Nipponbare IRSPG 1.0

193 genome using the HTSeq2, and the DESEq2 (similar to the iRAP) was used to identification of

194 differentially expressed genes (DEG) genes in response to $R$. solani infection in MCR and

195 CCDR varieties (cutoff adjusted P-value of $\leq=0.05$ ). Also, we extracted microarray expression

196 data for 14 SDRLK family genes from a published study (Narsai et al. 2013) describing

197 differential expression of genes in response to Xoo strain PX071 in the susceptible IR24 and

198 resistant IRBB21 rice cultivars. All extracted differential gene expression data for the rice

199

200

201

202

203

204

205

206

207

208

209

210

211

212

213

214

215

216

217

218 Results SDRLK genes under biotic stress conditions are available in Supplementary Table 4.

We extracted gene expression data for SDRLK family genes under abiotic stress conditions from E-MTAB-5941 (Buti et al. 2018), E-GEOD-38023 (Zhang et al. 2012), E-GEOD-41647, EMTAB-4994, E-MTAB-7317 and E-MTAB-3230 (Lima et al. 2015) datasets available at the EMBL-EBI Expression Atlas (processed similarly for differential gene expression and cutoff values $\log 2$-fold change 1.0 and adjusted $p \leq=0.05$ ) and have been analyzed by the iRAP pipeline. In addition, we extracted expression data from the supplementary tables associated with two peer-reviewed published articles (Hussain et al. 2016; Wu \& Yang 2020). All extracted differential gene expression data for the rice SDRLK family genes in response to abiotic stresses are available in Supplementary Table 5.

The gene expression data were visualized using Python scripts (available at https://github.com/naithanis/Naithani-lab-codes). The multicluster-SN_DK.py was used to generate gene cluster-based heatmaps for baseline tissue expression data. The

SN_DK_DiffX_cluster.py was used to visualize differential gene expression under biotic and abiotic stresses. The Venn diagrams were created online at http://bioinformatics.psb.ugent.be/cgi-bin/liste/Venn/calculate_venn.htpl.

219 


\section{SDRLKs family members have the variable gene and protein structures}

221 SRK gene, the prototype member of the SDRLK gene family, consists of 7 exons separated by

222 introns: the first exon encodes the entire extracellular S-domain, the second exon encodes the

223 transmembrane and juxta-membrane domains, and exons 3 to 7 encode the kinase domain (Stein

224 et al. 1991). Previously, the SDRLK family genes that have a conserved exon-intron structure

225

226

227

228

229

230

231

232

233

234

235

236

237

238

239

240

241

242

243

244

245

246

247

248

249

250

251

252

253

254

255

256

257

258

259 like SRK were grouped as SD1 type and genes having variable gene structures (some genes containing three introns, others containing one intron, and still others lacking introns) were grouped as the SD-2 and SD-3 types (Shiu \& Bleecker 2003; Shiu et al. 2004). The 144 rice SDRLK family genes include 53 SD1-type and 91 SD2-type genes. Out of 53 SD1 type genes, 49 contain one or more introns, and four lack introns. Out of 91 SD2 type genes, 77 lack introns, and 18 contain 1-3 introns (see Supplementary Table 1).

Previously, based on homology modeling, we have suggested that the $S$-domain of the $\mathrm{SRK}_{6}$ protein from Brassica oleracea consists of an N-terminal signal peptide (SP) and four structurally conserved domains, including two contiguous N-terminal Lectin-like domains (LLD) followed by an EGF-like domain, and a C-terminal PAN_APPLE domain (Naithani et al. 2007). Recently, the crystal structure of $\mathrm{SRK}_{9}$ bound to its cognate ligand the $\mathrm{SCR}_{9}$ confirmed this domain structure of SRK and further suggested that both N-terminal LLDs are globular and each consists of a nine-stranded $\beta$-barrel that forms a "Y"-shaped structure, the EGF-like domain contains an embedded short $\alpha$-helix, and the PAN_APPLE domain is highly similar to the Nterminal domain of hepatocyte growth factor (HGF) (Ma et al. 2016). Thus, the canonical structure for SRK includes SP, LLD1, LLD2, EGF-like, PAN_APPLE, TM, and Ser/Thr kinase domains (see Figure 1A). The UniProt database provides the basic prediction for all conserved domains and SLG sequence motif contained within LLD2 (Figure 1B) for SRK except for the EGF-like domain. Though prediction for highly polymorphic EGF-like domain is not consistent across various alleles of SRK, this region has six conserved cysteine residues in all SRKs and most SDRLK genes encoding full-length RLKs.

For comparison among SDRLK family proteins, we collected protein structural domain predictions from the UniProt protein knowledgebase (see Figure $1 \mathrm{C}$ ). Overall, this approach provides an approximate, but useful information on the structural comparisons among the SDRLK family members. If the structural information for individual proteins/gene models is available from published studies that was given a precedence over the predictions. As shown in Figure 1C, the SDRLK family proteins vary significantly in length, consisting of 67 full-length RLKs, 15 truncated RLKs, 14 S-domain (SD) kinases lacking a TM domains, 12 Receptor-like Proteins (RLPs) lacking kinase domains, and 36 kinases lacking any parts of the $S$-domain (including 10 kinases which have acquired one or more additional GnK2 domains). The detailed summary of predicted protein domains associated with the 144 rice SDRLK genes is provided in Supplementary Table 1.

\section{$50 \%$ of SDRLK family genes are tandem duplicates}

In the rice (O. sativa japonica Nipponbare) genome, most SDRLK family genes are located on

262

263 chromosomes 1 and 4 and 82 out of 144 SDRLK family genes are located alongside or nearby to other family members (Supplementary Figure 1). Considering variable structure and difference 
264 in the gene length, we built a sequence similarity tree to identify closely related genes (see

265 Supplementary Figure 2). Furthermore, we found that 72 (50\%) SDRLK family genes that are

266 highly similar to each other also share the same genomic neighborhood (see Supplementary

267 Figure 1 and 2), and thus qualified as being tandem duplicates. Our criteria for selecting tandem

268 duplicates ( $<$ five genes in between) are more restrictive than the criteria used in the other study

269 (Defoort et al. 2019), which consider genes found in the same genomic region within a

270 maximum of 30 genes apart as clusters of tandemly arranged genes. Our focus on a small

271 number of genes allowed us to conduct a more detailed evaluation of gene duplication events.

272 Overall, our analysis suggests that tandem gene duplication events have contributed significantly

273

274

275 Tissue-specific expression of SDRLK genes

276 We found baseline tissue-specific expression data for 112 rice (O. sativa ssp. japonica) SDRLK 277 genes in four datasets: E-GEOD-50777 (Anderson et al. 2013) contains data from the plant egg

278 cell, pollen sperm cell, and microgametophyte vegetative cell; E-MTAB-4123 (Zhang et al.

279 2014) has data from the shoot, anther, carpel, and seed; E-MTAB-2037 (Sakai et al. 2011)

280 contains data from the seed, callus, root, shoot, leaf, pre-flowering panicle, and post-flowering

281

282

283

284

285

286

287

288

289

290

291

292

293 panicle; and E-MTAB-2039 (Davidson et al. 2012) contains data from the leaf, early inflorescence, emerging inflorescence, anther, pistil, 5 day old seed, 10 days old seed, embryo, and endosperm (see Supplementary Table 2). We gathered additional information on expression for 15 additional rice SDRLK family members (see Supplementary Table 3) from published literature (Aya et al. 2011; Chen et al. 2013; Chen et al. 2006; Fan et al. 2018; Gao \& Xue 2012; Li et al. 2018; Lin et al. 2019; Pan et al. 2020; Zhai et al. 2013; Zou et al. 2015). The SDRLK family genes show varied expression profiles: 25 genes show a unique tissue-specific expression profile, 44 genes show expression in more than one tissues, and 58 ubiquitously expressed genes show preferential expression in one or more tissues (see Figure 2 and Supplementary Table 6). Overall, 86 genes express in the leaf, 83 genes express in the root, 103 genes express in the flower, 87 express in the seed, and 82 express in the shoot (Supplementary Figure 3 ). We found expression data for eight genes in seedlings or whole plants in the differential gene expression datasets used in this study and no information for nine genes (see Supplementary Table 6).

\section{SDRLK family genes are regulated in response to biotic stresses}

295

296

297

298

299

300

\section{Bacterial blight response}

302

303

304

305 section.
We analyzed the changes in gene expression of rice SDRLK family members in response to bacterial, viral, and fungal pathogens using publicly available transcriptomic datasets. A summary of differentially expressed genes (DEG) in response to various biotic agent is provided in the Supplementary Table 7. Details about datasets used in this study are described in Methods

We found two micro-array datasets E-GEOD-61833 (Girija et al. 2017), and E-GEOD-36272 in the EMBL-EBI Expression Atlas, and one published study (Narsai et al. 2013) containing expression data (see Supplementary Table 4) for SDRLK family members in response to bacterial pathogen $X o o$ (the causal agent of bacterial blight disease in rice). 
306

307

308

309

310

311

312

313

314

315

316

317

318

319

320

321

322

323

324

325

326

327

328

329

330

331

332

333

334

335

336

337

338

339

340

341

342

343

344

345

346

347

348

349

350

351
E-GEOD-61833 contains differential expression data in response to three Xoo strains: BXO43 (wildtype), BXO1002 exopolysaccaride deficient (EPS-), and BXO1003 (LPS-, EPS-) double mutant deficient in both EPS and lipopolysaccharide (LPS) (Girija et al. 2017). LPS is a component of the bacterial cell wall. Following infection, LPS is recognized by pattern recognition receptors (PRRs) located in the plasma membrane of the rice cells as 'Microbe Associated Molecular Patterns' (MAMPs) that in turn, activates plant triggered immunity (PTI). PTI, a broad-spectrum defense response, provides basal resistance. To counter host PTI, bacterial pathogens rely on virulent factors and the type III secretion system (T3SS). The T3SS secretes multiple effector proteins into host cells (He et al., 2004) including EPS to modulate the host's gene expression and promotes infection (Girija et al. 2017). This dataset provides an insight into interactions between counteractive mechanisms, immunity, and infection. As shown in the Figure 3A, ten genes, including Os01g0668901, Os01g0783800 (SDS2), Os04g0103700, Os04g0356600, Os04g0419900, Os04g0631800, Os05g0165900, Os07g0534500, Os09g0454900, and Os12g0527700 show higher expression in strain BXO1002 (LPS $\left.{ }^{+} \mathrm{EPS}^{-}\right)$ compared to both wild-type (LPS ${ }^{+} \mathrm{EPS}^{+}$) and double mutant BXO1003 (LPS-, EPS-) suggesting their role in LPS-induced and EPS-suppressed PTI. In contrast, three genes, Os01g0669100, Os01g0670100, and Os10g0101000, show higher expression in both BXO1002 (LPS EPS$^{-}$) and BXO1003 (LPS-, EPS-) compared to BXO43, and thus, likely to be independent of LPS induced PTI, but down-regulated by EPS-mediated signaling. Os02g0472700 and Os04g0226600 express at similar levels in all three strains and are likely to be independent of LPS and EPS signaling.

The E-GEOD-36272 contains differential expression data for 41 SDRLK family members from susceptible rice varieties IR24 (O. sativa indica IR24) and Nipponbare (O. sativa japonica Nipponbare) in response to nine $X$. oryzae strains. The $X$. oryzae pv. oryzicola $(X o c)$ strain BLS303 causes bacterial streak diseases and not blight. The wildtype Xoo strains T7174, PX086, PX099A-2, and PX099A are pathogenic and cause blight disease. PXO99AME7 is a nonpathogenic strain (lacking a functional T3SS), and PXO99 ${ }^{\mathrm{A}} \mathrm{ME5}$ (unknown mutation), PXO99 ${ }^{\mathrm{A}} \mathrm{ME} 2$ ( $p$ thXo1), PXO99AME1 (pthXo6 and $\left.a v r X a 27\right)$ are low virulent strains containing mutations in the transcription activator-like (TAL) genes. The pthXo1 is a major TAL effector gene in the PX099A that promotes disease in rice (host) cells. AvrXa27, another effector from Xoo activates transcription of a disease-resistant gene Xa27 in rice. The loss of pthXol (in PXO99A ME2) or pthXo6, and $a v r X a 27$ (in PXO99A ME1) results in low virulence. This dataset can be used for identifying genes specifically regulated in response to PXO99A in susceptible rice varieties IR24 and Nipponbare. Figure 3B shows the expression profile of 41 genes in IR24 and Nipponbare. Interestingly, Os01g0668901 and Os10g0342300, both encoding full-length RLKs, are upregulated in IR24 and Nipponbare in response to Xoo wildtype strains, but not in response to T3SS defective mutant or TAL mutants suggesting their role in pthXol mediated effector-triggered immunity (ETI). Os01g0668901 is highly expressed in reproductive cells (pollen sperm cell, egg cell, and embryo). However, Os10g0342300 shows preferential expression in root and flower but also expresses in leaf, shoot, and seeds (Aya et al. 2011). Os05g0166300, Os05g0165900, and Os01g0669100 are generally upregulated in response Xoo in Nipponbare, but seems to be independent of the T3SS derived effectors (likely to be part of PTI). Os04g0419700 shows up-regulation only in the IR24 genotype in response to PXO99AME1 and PXO99AME2, suggesting its down-regulation by pthXo1. Os06g0165500 and Os08g0179000 are likely to be down-regulated by pthXol in IR24 in response to PX099A. 
352 Os04g0420033 is specifically down-regulated in Nipponbare in response to all Xoo strains and

353

354

355

356

357

358

359

360

361

362

363

364

365

366

367

368

369

370

371

372

373

374

375

376

377

378

379

380

381

382

383

384

385

386

387

388

389

390

391

392

393

394

395

likely to be up-regulated by avrXa27. In response to Xoc strain BLS303, five genes

(Os01g0668600, Os01g0670100, Os02g0710500, Os04g0632600, and Os06g0602500) in IR24

and seven genes (Os01g0890600, Os04g0226600, Os04g0631800, Os04g0632600,

Os05g0165900, Os07g0534500, Os10g0136400, and Os10g0136500) in Nipponbare show

differential expression, however, only Os04g0632600 is common in both cultivars.

The third dataset, extracted from Narsai et al. (2013), contains expression data for 14 SDRLK family genes from a susceptible rice cultivar IR24, and a resistant cultivar IRBB21 in response to Xoo strain PX071 infection. As shown in Figure 3C, ten genes are up-regulated in resistant IRBB21 (but not in susceptible IR24) in response to PXO71: one gene Os10g0136400 shows upregulation during 24 hours post-infection (early), five genes (Os03g0838100, Os04g0632600, Os06g0689600, Os07g0534500, and Os10g0136500) show up-regulation during 96 hours postinfection (late), and four genes (Os04g0226600, Os04g0655300, Os08g0343000, and

Os06g0602500) show up-regulation early on and their expression is maintained in the late phase. Two genes Os01g0784700 and Os07g0551300 are down-regulated only in resistant IRBB21during the late phase. Also, Os01g0670300 is down-regulated and Os12g0527700 is upregulated only in the susceptible IR24.

In the E-GEOD-36272, we found data for 41 SDRLK family members that includes 16 out of 18 genes in E-GEOD-61833. The missing two genes Os07g0550500 and Os10g0101000 from EGEOD-61833 show expression only in response to the EPS- strains. Further, eight out of fourteen genes from Narsai et al. (2013) are common with E-GEOD-36272 and remaining 6 genes (Os01g0670300, Os03g0838100, Os04g0655300, Os06g0689600, Os07g0551300, and Os08g0343000) are unique. Total 49 rice SDRLK family genes show differential expression in response to Xoo. The presence of common DEG across three independent studies strongly suggests their role in Xoo pathogenesis and plant immunity.

\section{Bacterial panicle blight response}

B. glumae causes bacterial panicle blight disease of rice that is characterized by having florets with a darker base and a reddish-brown margin, and frequently upright due to poor filling (Magbanua et al. 2014; Nandakumar et al. 2009). This disease also causes sheath rot and seedling blight. E-MTAB-4406 RNA-Seq dataset (Magbanua et al. 2014) contains data for 72 SDRLKs family genes (see Supplementary Table 4) that was generated from seedlings and inflorescence of a moderately resistant rice genotype CL 161 and a susceptible rice genotype CL 151 infected with B. glumae pathogenic strain $89 \mathrm{gr}-4$. Figure 4 shows 18 DEG when resistant CL 161 genotype is compared with the susceptible CL 151 genotype in response to B. glumae infection. Five genes (Os01g0222800, Os03g0221700, Os04g0201900, Os04g0633200, and Os12g0130200) show up-regulation, and two genes (Os03g0556600 and Os04g0475200) show down-regulation at the flowering stage in CL 161 in comparison to CL 151. Three genes (Os01g0889900, Os06g0689600 and Os1 1g0133001) show up-regulation at seedling as well as the flowering stage in CL 161 in comparison to CL 151. Six genes (Os01g0113350, Os01g0155200, Os01g0223900, Os01g0668600, Os09g0551400, and Os11g0441900) are upregulated in CL 161 compared to CL 151 at the seedlings stage. Os07g0534700 and

Peer) reviewing PDF | (2020:07:51145:3:0:NEW 25 Jan 2021) 
396 Os07g0550900 are down-regulated at the seedling stage but up-regulated at flowering stage in

397 CL 161 compared to CL 151.

\section{Stripe disease response}

399 Rice stripe virus (RSV), a single-stranded RNA virus, causes stripe disease in rice. This virus is

400 transmitted through brown plant hoppers Laodelphax striatellus only, and direct plant to plant 401 transmission of the virus doesn't occur. E-MTAB-5025 dataset contains expression data from 402 rice plants infected using viruliferous insect vector $L$. striatellus or by microinjection containing 403 insect- / plant-derived RSV(Zhao et al. 2016). It was demonstrated that plants inoculated with 404 plant-derived RSV using microinjection failed to cause stripe symptoms, whereas plants directly 405 infected by insect vectors or microinjected with insect-derived RSV showed symptoms of the 406 disease suggesting that the RSV acquires pathogenicity during its replication inside the insect 407 vector (Zhao et al. 2016). We found expression data for 41 SDRLK family genes in E-MTAB4085025 (see Supplementary Table 4). As shown in Figure 5A, no significant change in gene 409 expression was observed when plant-derived RSV was microinjected into healthy plants. In

\section{Leaf-sheath blight response}

420

421

422

423

424

425

426

427

428

429

430

431

432

433

434

435

436

437 contrast, plants infected with insect-derived RSV (microinjected or delivered via viruliferous insect vector) share five down-regulated genes (Os01g0890600, Os11g0133100, Os12g0130200, Os12g0130300, and Os12g0527700) and seven up-regulated genes (Os01g0224000, Os02g0710500, Os03g0556600, Os04g0103500, Os04g0420900, Os07g0550900, and Os09g0454900). This is consistent with the previous observation that in both categories of plants show typical stripe disease symptoms and damage to chloroplast structure (Zhao et al. 2016). Further experimental analysis will be required for deciphering the role of these twelve genes in rice stripe disease development/resistance.

The fungal pathogen Rhizoctonia solani causes leaf sheath blight disease of rice. E-MTAB-6402 (ERS2106879-ERS2106902) dataset contains expression data for 39 SDRLK family genes (see Supplementary Table 4) from a resistant rice genotype MCR010277 (MCR), and a susceptible rice Cocodrie (CCDR) infected with $R$. solani strain LR172 for 3 days (early) and 5 days (late). Both CCDR and MCR are long-grain, high-yielding breeding line developed in the U.S (https://www.lsuagcenter.com). As shown in Figure 5B, six genes (Os01g0668901, Os01g0784700, Os01g0889900, Os02g0472700, Os02g0710500, and Os07g0534700) show upregulation and four genes (Os01g0668400, Os04g0201900, Os04g0631800, and Os04g0632901) show down-regulation in resistant MCR at 3 Days Post Infection (DPI) but not in susceptible CCDR. Os07g0550500 shows up-regulation at 3 DPI as well as at 5 DPI in the resistant MCR but not in CCDR. No change in gene expression is observed in CCDR 5-days post-infection. Three genes Os01g0670100, Os01g0890600, and Os06g0689600 show increased expression in CCDR at 3-days post-infection compared to MCR. In total, 14 rice SDRLK family genes show differential expression in response to $R$. solani.

\section{SDRLKs are regulated in response to abiotic stresses}

We analyzed the changes in gene expression of rice SDRLK family members in response to various abiotic stresses using publicly available transcriptomic datasets (see Supplementary

Peer] reviewing PDF | (2020:07:51145:3:0:NEW 25 Jan 2021) 
438 Table 5 and Methods for details). A summary of genes involved in one or more abiotic stress

439

440

441

442

443

444

445

446

447

448

449

450

451

452

453

454

455

456

457

458

459

460

461

462

463

464

465

466

467

468

469

470

471

472

473

474

475

476

477

478

479

480

481 response is provided in Supplementary Table 7.

\section{Chilling response}

Rice is sensitive to chilling stress, especially at the seedling stage, which negatively impacts the plant's growth and yield. To understand SDRLK family members' role in chilling/cold tolerance and susceptibility, we analyzed two datasets E-MTAB-5941 (Buti et al. 2018) and E-GEOD38023 (Zhang et al. 2012). We find 37 genes common to both datasets; 18 unique in E-MTAB5941; and 22 unique in the E-GEOD-38023 (total 77 genes).

E-MTAB-5941 RNA-seq dataset provides an opportunity to compare the short- and long-term stress-induced changes in the transcriptome of a chilling-sensitive genotype Thaibonnet and a chilling-tolerant genotype Volano, each subjected to 2 and 10 hours chilling treatment at $10^{\circ} \mathrm{C}$ (Buti et al. 2018). As shown in Figure 6A, overall, 55 SDRLK genes show differential expression in response to chilling stress in the two genotypes. In comparison to Thaibonnet, the Volano shows up-regulation of four genes (Os05g0166300, Os04g0419900, Os06g0164900, and Os04g0632100) in response to short-term chilling stress; up-regulation of four genes (Os07g0534500, Os01g0669100, Os06g0602500 and Os07g0534700) in response to long-term chilling stress; and up-regulation of Os07g0535800 in both short- and long-term chilling stress. Also, six genes (Os10g0136500, Os06g0541600, Os10g0136400, Os04g0633300, Os01g0890600 and Os12g0527700) show decreased expression in the tolerant Volano compared to the susceptible Thaibonnet. Overall, 15 DEGs from this subfamily are likely to play a role in the chilling tolerance gene-networks.

E-GEOD-38023 contains expression data from a chilling-tolerant Li-Jiang-Xin-Tuan-He-Gu (LTH) japonica landrace variety and chilling-sensitive IR29 indica cultivar. The plants from both genotypes were subjected to chilling treatment at $4^{\circ} \mathrm{C}$ for $2,8,24$, and 48 hours, and then moved to normal temperature $29^{\circ} \mathrm{C}$ for 24 hours to allow recovery (Zhang et al. 2012). We found expression data for 59 SDRLK genes in this dataset (see Supplementary Table 5). As shown in Figure 6B, under chilling stress, many common genes are up-regulated in both genotypes at an early stage. But, at later stages, we find up-regulation of genes in the chilling-tolerant LTH and strong repression in the chilling-sensitive IR29. Specifically, in LTH genotype, eight genes show up-regulation (early response genes Os01g0224000, Os10g0101000; and late response genes Os04g0632600, Os03g0556600, Os12g0640700, Os01g0155200, Os07g0534700, Os04g0420900), and three genes (Os05g0166300, Os01g0889900, and Os01g0223900) show down-regulation in comparison to IR29. Also, five genes (Os04g0419900, Os06g0602500, Os03g0422800, Os06g0690200, and Os01g0885700) show increased expression in LTH in response to chilling at early stage and their expression is maintained in the late phase (Figure 6B). Overall, 16 SDRLK family genes are differentially regulated in response to chilling between the tolerant and the susceptible rice genotypes.

\section{Drought response}

We found drought-induced differential gene expression data for 59 SDRLK family genes from three microarray datasets (E-GEOD-41647, E-MTAB-4994, and E-MTAB-3230), and one RNA- 
482

483

484

485

486

487

488

489

490

491

492

493

494

495

496

497

498

499

500

501

502

503

504

505

506

507

508

509

510

511

512

513

514

515

516

517

518

519

520

\section{1}

522

523

524

525

526

seq dataset E-MTAB-7317 (see Supplementary Table 5). The data from four datasets were compiled together for visualization (direct comparison across datasets were not made, but a summary is given in Supplementary Table 7).

The E-GEOD-41647 contains data for 26 SDRLK family genes from seedlings of susceptible IR20 and drought-tolerant Dagad deshi genotypes. We find five genes (Os01g0223700, Os01g0669100, Os03g0422800, Os06g0575400, and Os08g0179000) are down-regulated and ten genes (Os04g0420900, Os04g0632100, Os09g0454900, Os04g0632600, Os06g0689600, Os06g0690200, Os06g0241100, Os06g0602500, Os01g0224000, and Os01g0885700) are upregulated in the Dagad deshi but not in the IR24 (see Figure 7). Thus, 15/26 genes from this set appear are likely to play a role in the drought response pathways. The E-MTAB-4994 contains data for 20 SDRLK subfamily genes from the flag leaf at the panicle initiation stage of Oryza sativa Indica cultivar Nagina 22 (a drought-tolerant genotype). As shown in Figure 7, ten genes show up-regulation in response to drought in Nagina 22. E-MTAB-3230 contains data for eight genes from seedlings of Nagina 22 and an enhanced drought-tolerant mutant ewst 1 of Nagina 22 grown in hydroponic culture subjected to $25 \%$ polyethylene glycol (PEG) for one hour (Lima et al. 2015) to mimic drought stress. We find that six genes are down-regulated and two genes are up-regulated in ewst1 mutant compared to Nagina 22 in response to PEG treatment. The two upregulated genes Os07g0550900 and Os07g0551300 are unique to the ewst 1 mutant (see Figure 7).

E-MTAB-7317 contains data for 44 SDRLK family genes from drought tolerant Nagina 22 and a salt-tolerant genotype Nonabokra (drought tolerance unknown), each subjected to drought for 2days (early) and 3-days (late). As shown in Figure 7, many SDRLK family members show similar expression patterns between Nagina 22 and Nonabokra, suggesting that the latter might possess some level of drought tolerance. However, in comparison to Nonabokra, Nagina 22 shows a significant difference in the drought response: eight genes (Os01g0223800, Os01g0670100, Os01g0890600, Os04g0420033, Os05g0166300, Os07g0534500, Os07g0550500, and Os12g0527700) are down-regulated, and seven genes (Os01g0222800, Os01g0366300, Os04g0632100, Os04g0633600, Os04g0634400, Os12g0130300, and Os12g0130800) are up-regulated in Nagina 22 but not in Nonabokra. Also, Os06g0165500 is upregulated early in Nagina 22, whereas in Nonabokra it is up-regulated during the late phase.

Overall, three drought-tolerant genotypes, Dagad deshi, Nagina 22, and ewst1_Nagina 22 share four common up-regulated genes (Os01g0224000, Os04g0632100, Os06g0690̄200, and Os09g0454900) and four common down-regulated genes (Os01g0223700, Os01g0669100, Os03g0422800, and Os08g0179000) in response to drought suggesting their conserved role in drought response.

\section{Submergence response}

Rice (Oryza sativa) is unique among cereals that can germinate under submerged conditions and tolerate an anoxic environment. The ability to withstand short-and long-term submergence stress varies widely among the rice cultivars and is crucial to their adaptation to a range of geographical regions, particularly the world's coastal regions. Considering RLKs play roles in cell signaling, we searched for gene expression data for SDRLK family genes under

Peer] reviewing PDF | (2020:07:51145:3:0:NEW 25 Jan 2021) 
527 submergence stress and found data for 35 genes in the supplementary tables associated with two

528 recent publications (Hussain et al. 2016; Wu \& Yang 2020) (see Supplementary Table 5).

529

530

531

Wu and Yang (2020) investigated the role of auxin signaling in the coleoptile growth of Oryza sativa L. japonica cultivar Taikeng 9 seedlings under submergence and showed that a polar

532

533

534

535

536

537

538

539 auxin transport inhibitor 2,3,5-triiodobenzoic acid (TIBA) caused delay in seeds germination and reduced growth of the coleoptile under submergence. Furthermore, the authors identified 3448 up-regulated and 4360 down-regulated genes in response to submergence and TIBA treatment. We found gene expression data for 20 SDRLK family genes in this dataset. As shown in Figure 8, five genes (Os04g0103700, Os07g0535800, Os02g0710500, Os01g0587400, and Os05g0166300) show down-regulation, and fifteen genes show up-regulation in the submerged seedling supplied with TIBA in comparison to submerged seedling (without TIBA). These results suggest the association of these SDRLK genes with submergence response and auxinmediated signaling required for coleoptile growth.

541

542

543

544

545

546

547

548

549

550

Hussain et al. (2016) studied the protective effects of seed priming under submergence in Indica inbred Oryza sativa L. cultivar Huanghuazhan by comparing transcriptomes of 4-day-old seedlings. The samples included submerged seedlings grown from non-primed and primed seeds with selenium (Se) or salicylic acid (SA). In this dataset, we found nineteen SDRLK family genes showing differential expression (see Supplementary Table 5). The Se-priming of rice seeds prior to submergence modified the expression of four genes (Os06g0496800, Os01g0669100, Os03g0221700, and Os04g0419900) when compared to non-primed (NP) submerged plants, and mimic the control plants not subjected to submergence stress. Similarly, the SA-priming modified the expression pattern of Os06g0241100, Os07g0141100, Os02g0767400, and Os06g0496800 in comparison to and non-primed (NP) submerged plants (see Figure 8). In both, SA- and Se-primed seedlings Os06g0496800 is up-regulated to mitigate the effect of submergence. In addition, Os12g0130300 shows increased expression in SA- and Se-primed seedlings but no differential regulation in response to submergence, suggesting that this gene may be affected by the materials or methods used for seed priming. Notably, four genes Os06g0241100, Os06g0575000, Os07g0141100, and Os05g0166300 up-regulated under submergence in this dataset are in common with the dataset from Wu and Yang (2020), suggesting their conserved function in both rice cultivars.

In total, 115 SDRLK family genes show differential expression in response to environmental stimuli, including eight genes not expressed under normal growth conditions. Specifically, 36 genes are significantly regulated under abiotic stress conditions in tolerant genotypes in comparison to susceptible genotypes; 17 genes are significantly regulated under biotic stresses in resistant genotypes in comparison to susceptible genotypes; and 34 genes regulated in response

566 to both biotic and abiotic stress conditions between tolerant and susceptible genotypes (see Supplementary Table 7). We find 81 common genes regulated in response to both biotic and abiotic stress conditions also express under normal growth and developmental conditions (Supplementary Figure 3). Notably, a few genes expressed in the seed under normal growth conditions are highly expressed in leaf and other vegetative tissue under stress. For example, Os05g0501400 expresses only in seed under normal growth condition, but expresses in seedlings 
572 under submergence; Os09g0551400 shows low expression in seed but up-regulated seedlings of

573 resistant genotype CL 161 in response to $B$. glumae infection. This is consistent with the

574 previous observation that many genes involved in the seed development are also regulated under

575 stress (Naithani et al. 2017).

576

\section{Discussion}

578 The rice SDRLK subfamily of receptor-like kinases includes 144 members, including full-length

579 gene duplicates, partial gene duplicates, and pseudogenes. 72 (50\%) genes of the rice SDRLK

580 family are tandem duplicates, and the majority is in chromosomes 1 and 4 . In general, the

581 expansion and diversification of the SDRLK gene family in rice results from the tandem gene

582 duplication of full-length or partial gene fragments, recombination, and ancient whole-genome

583 duplications. This is consistent with this family's evolution in other plant species (Lehti-Shiu et

584 al. 2009; Myburg et al. 2014; Shiu \& Bleecker 2003). This gene subfamily was named after

585 SRK, the female determinant of SI response in the Brassica and most of its members (full-length

586 RLKs) bear gene annotation "S-locus Receptor-like Kinase' involved in 'self-incompatibility

587 response'. These gene annotations were assigned by automated gene-orthology and Gene

588 Ontology pipelines currently being used for large-scale gene annotation of sequenced genomes.

589 Other genes of the family that are partial gene duplicates are assigned 'unknown function' (see

590 Supplementary Table 1). The automated projections of gene annotations from the well-

591 characterized orthologs in other organisms is a powerful approach for identifying highly

592 conserved genes and for preliminary gene annotations at the genome-scale. However, it falls

593 short in assigning correct gene annotations and functional associations to the individual members

594 of large gene families. Often, most members of a gene family or its subclass bear similar gene

595 annotation without considering the evolutionary fate of gene duplicates, such as sub-

596 functionalization, functional diversification, neo-functionalization, and pseudogenization.

597 Furthermore, rice (O. sativa) is a self-fertilizing and the sporophytic self-incompatibility does not

598 exist in this species. Considering SDRLK gene family's size, inaccurate gene-annotations and

599 functional associations impacts all types of large-scale genomic and transcriptomic analyses.

600 Thus, a manual biocuration approach is needed for correcting gene annotations of the SDRLK

601 gene family.

602

603

To date, the functions of most SDRLKs are unknown, however, it is increasingly becoming clear

604 that these genes are expressed in a variety of tissues and in response to both biotic and abiotic

605

606

607

608

609

610

611

612

613

614

615

616

617 stresses (Lehti-Shiu et al. 2009; Vining et al. 2015) and play crucial roles in many important plant processes beyond self-incompatibility, such as in root development (Pan et al. 2020), leaf senescence (Chen et al. 2013), stem elongation ( $\mathrm{Li}$ et al. 2018), in grain yield, and pathogen resistance in rice (Kim et al. 2009; Wang et al. 2015; Zou et al. 2015). Moreover, we have vast amount of publicly available transcriptomic data that can reveal new information about the expression of the SDRLK family genes and their associations with important biological processes. We utilized publicly available genomic and transcriptomic data to study the structure of rice SDRLK subfamily genes and their expression across various tissues under normal growth and developmental conditions as well as in response to pathogen infection and abiotic stresses; and conducted a thorough review of the published literature to gather additional information. We found expression information for 135 rice SDRLK family genes. 128 rice SDRLK family genes are expressed under normal growth and developmental conditions, including 27 genes showing unique tissue-specific expression, 43 genes showing preferential expression in more than one 
618 tissue, 58 genes showing ubiquitous expression but varying in degree of expression across tissue

619 types (see Figure 3 and Supplementary Table 6). Furthermore, 36 SDRLK genes that are

620 expressed under normal growth are not regulated in response to any stress condition. However,

621 tissue-specific expression patterns suggest their potential role in plant development and/or

622 formation of specific organs/tissues/cell types. One gene from this group, PSRK2, controls stem

623

624

625

626

627

628

629

630

631

632

633 elongation by negatively regulating GA responses (Li et al. 2018).

Overall, 99 SDRLK family genes show differential expression in response to environmental stimuli, including seven genes not expressed under normal growth conditions (Supplementary Table 7). Specifically, 39 genes are regulated under abiotic stress conditions; 23 genes regulated under biotic stresses; and 37 genes regulated in response to both biotic and abiotic stress conditions. In general, we find that most SDRLK family genes respond to more than one stress condition, which seems to be consistent with the role of many kinases in cell-signaling. In general, we find overlapping expression patterns in many tandem duplicates and identical tissuespecific expression profiles for ten pairs of tandem duplicates (highlighted in bold in

634

635

636

637 Supplementary Table 6) and identical profiles of four pairs in response to stress treatment (highlighted in bold in Supplementary Table 7). Notably, Os08g0179000 shows flower-specific, and Os08g0179150 shows preferential expression in flower; however, both genes are predicted to encode two complementary halves of an RLK, and correspond to one gene LOC_Os08g08140 in the MSU database. Gene models for both genes are likely incorrect in the RAP database, and a correction is needed.

639

640

641

Based on a careful evaluation, we have created a list of candidate genes that are likely to play an important role in conferring tolerance to one or more abiotic stresses and/or resistance to

642

643 pathogens (Supplementary Table 7). A few genes from this family have been experimentally characterized in detail and their role in conferring biotic resistance and abiotic stress tolerance has been confirmed. In particular, Os06g0494100 (PID2) confers race-specific resistance to $M$.

646 grisea (Wang et al. 2015); Os01g0783800 (SDS2) controls programmed cell death and provides resistance to Magnaporthe oryzae (Fan et al. 2018); Os01g0223800 (ESG1), induced by

647 treatment with $\mathrm{PEG}, \mathrm{NaCl}$, abscisic acid (ABA) and drought, provides drought tolerance (Pan et al. 2020); and Os07g0186200 (SIK2), also induced by NaCl, drought, cold, dark, and ABA, is involved in drought and salt tolerance, and delays dark-Induced leaf senescence (Chen et al. 2013). Also, Os01g0669100 (LSK1) controls branching per panicle and grain yield (Zou et al. 2015). We expect that many genes listed in Supplementary Table 7 are likely to play a role in mitigating one or more stresses and can be exploited for breeding advanced high-yielding rice varieties. Some SDRLK family genes have the potential for direct applications in improving and managing crop productivity. For instance, the submergence tolerance traits can also be exploited commercially to manage weeds in paddy fields, where controlled flooding would allow the germination of rice seeds and seedlings' growth but suppress the weeds. However, further functional assays and phenotypic studies on cultivated and wild rice species will be required on these candidate loci/genes to ascertain their exact function.

659

660

We aim to improve and enrich the gene description in public databases and help the plant 661 research community identify potential candidate genes. Here, we re-used and re-analyzed 662 publicly data to synthesize new information about the structure and function of SDRLK gene 663 family members. Based on the analysis of gene structure, transcription profile, and extensive 
664 literature review, we propose the gene nomenclature and gene description for the 144 members 665 of the rice SDRLK family (see Supplementary Table 1). We will integrate this information in the 666 Plant Reactome knowledgebase (Naithani et al. 2020) that also exchanges information with other public resources such as UniProt and the RAP database.

In addition to retrieving new knowledge and enriching public databases, manual biocuration projects teach students/researchers genomic data literacy, help to build the community of biocurators (Naithani et al. 2019), and provide peer-review of the public genomic datasets. The

672 re-use of genomic and transcriptomic data for mining new insights ('data parasitism') is already making significant impact in the field of genomics and the value of secondary data analyses and

674 meta-analyses is being recognized. The data re-use is one of the core component of FAIR data principle. We expect that our study sets forth an example of re-use and re-analysis of genomic data for improving gene annotation of the large gene families and enriching the contents of public genomic databases and resources via community biocuration efforts.

\section{Conclusions}

681

Plant genomes harbor extensive gene duplications and large gene families consisting of members

682 ranging from redundant gene duplicates, gene duplicates showing tissue- and developmental stage-specific function, genes expressed only under stress conditions, genes with new

683

684 /diversified functions, and pseudogenes. However, the individual members of the gene families are poorly annotated in the public databases. Thus, researchers often ignore these genes as potential candidate genes for further experimental analysis. We utilized publicly available genomic and transcriptomic data to improve gene annotation and descriptions of rice SDRLK subfamily genes and identified potential candidate genes likely to play a role in conferring tolerance to one or more biotic and abiotic stresses. We see tremendous potential in re-analysis of publicly available genomic data to improve the annotations of the members of large gene families, build gene-gene interaction networks, and identify candidate genes likely to impart important traits related to yield and climate resilience. Manual biocuration of genes by researchers can improve the contents of genomic databases and complement high-throughput automated protocols.

\section{Acknowledgments}

We acknowledge Justin Preece (Jaiswal lab, Oregon State University) for helping DD with

697 Python scripting.

698

\section{Conflict of interest:}

700 $\mathrm{PJ}$ is an academic editor for the PeerJ journal.

701

702 


\section{References}

704

705

706

707

708

709

710

711

712

713

714

715

716

717

718

719

720

721

722

723

724

725

726

727

728

729

730

731

732

733

734

735

736

737

738

739

740

741

742

743

744

745

746

747

748

749

750

751

752

Ahmadi B, Masoomi-Aladizgeh F, Shariatpanahi ME, Azadi P, and Keshavarz-Alizadeh M. 2016. Molecular characterization and expression analysis of SERK1 and SERK2 in Brassica napus L.: implication for microspore embryogenesis and plant regeneration. Plant Cell Reports 35:185-193. 10.1007/s00299-015-1878-6

Anderson SN, Johnson CS, Jones DS, Conrad LJ, Gou X, Russell SD, and Sundaresan V. 2013. Transcriptomes of isolated Oryza sativa gametes characterized by deep sequencing: evidence for distinct sex-dependent chromatin and epigenetic states before fertilization. Plant Journal 76:729-741. 10.1111/tpj.12336

Aya K, Suzuki G, Suwabe K, Hobo T, Takahashi H, Shiono K, Yano K, Tsutsumi N, Nakazono M, Nagamura Y, Matsuoka M, and Watanabe M. 2011. Comprehensive network analysis of anther-expressed genes in rice by the combination of 33 laser microdissection and 143 spatiotemporal microarrays. PLoS ONE 6:e26162. 10.1371/journal.pone.0026162

Becraft PW, Stinard PS, and McCarty DR. 1996. CRINKLY4: A TNFR-like receptor kinase involved in maize epidermal differentiation. Science 273:1406-1409. 10.1126/science.273.5280.1406

Berckmans B, Kirschner G, Gerlitz N, Stadler R, and Simon R. 2020. CLE40 Signaling Regulates Root Stem Cell Fate. Plant Physiology 182:1776-1792. 10.1104/pp.19.00914

Boggs NA, Dwyer KG, Shah P, McCulloch AA, Bechsgaard J, Schierup MH, Nasrallah ME, and Nasrallah JB. 2009. Expression of distinct self-incompatibility specificities in Arabidopsis thaliana. Genetics 182:1313-1321. 10.1534/genetics.109.102442

Buti M, Pasquariello M, Ronga D, Milc JA, Pecchioni N, Ho VT, Pucciariello C, Perata P, and Francia E. 2018. Transcriptome profiling of short-term response to chilling stress in tolerant and sensitive Oryza sativa ssp. Japonica seedlings. Functional \& Integrative Genomics 18:627-644. 10.1007/s10142-018-0615-y

Cartwright HN, Humphries JA, and Smith LG. 2009. PAN1: a receptor-like protein that promotes polarization of an asymmetric cell division in maize. Science 323:649-651. 10.1126/science.1161686

Chen LJ, Wuriyanghan H, Zhang YQ, Duan KX, Chen HW, Li QT, Lu X, He SJ, Ma B, Zhang WK, Lin Q, Chen SY, and Zhang JS. 2013. An S-domain receptor-like kinase, OsSIK2, confers abiotic stress tolerance and delays dark-induced leaf senescence in rice. Plant Physiology 163:1752-1765. 10.1104/pp.113.224881

Chen X, Shang J, Chen D, Lei C, Zou Y, Zhai W, Liu G, Xu J, Ling Z, Cao G, Ma B, Wang Y, Zhao X, Li S, and Zhu L. 2006. A B-lectin receptor kinase gene conferring rice blast resistance. Plant Journal 46:794-804. 10.1111/j.1365-313X.2006.02739.x

Clark SE, Williams RW, and Meyerowitz EM. 1997. The CLAVATA1 gene encodes a putative receptor kinase that controls shoot and floral meristem size in Arabidopsis. Cell 89:575585. 10.1016/s0092-8674(00)80239-1

Davidson RM, Gowda M, Moghe G, Lin H, Vaillancourt B, Shiu SH, Jiang N, and Robin Buell C. 2012. Comparative transcriptomics of three Poaceae species reveals patterns of gene expression evolution. Plant Journal 71:492-502. 10.1111/j.1365-313X.2012.05005.x

Defoort J, Van de Peer Y, and Carretero-Paulet L. 2019. The Evolution of Gene Duplicates in Angiosperms and the Impact of Protein-Protein Interactions and the Mechanism of Duplication. Genome Biol Evol 11:2292-2305. 10.1093/gbe/evz156

Escobar-Restrepo JM, Huck N, Kessler S, Gagliardini V, Gheyselinck J, Yang WC, and Grossniklaus U. 2007. The FERONIA receptor-like kinase mediates male-female interactions during pollen tube reception. Science 317:656-660. 10.1126/science.1143562

Fan J, Bai P, Ning Y, Wang J, Shi X, Xiong Y, Zhang K, He F, Zhang C, Wang R, Meng X, Zhou J, Wang M, Shirsekar G, Park CH, Bellizzi M, Liu W, Jeon JS, Xia Y, Shan L, and Wang

Peer) reviewing PDF | (2020:07:51145:3:0:NEW 25 Jan 2021) 
753

754

755

756

757

758

759

760

761

762

763

764

765

766

767

768

769

770

771

772

773

774

775

776

777

778

779

780

781

782

783

784

785

786

787

788

789

790

791

792

793

794

795

796

797

798

799

800

801

802

803
GL. 2018. The Monocot-Specific Receptor-like Kinase SDS2 Controls Cell Death and Immunity in Rice. Cell Host Microbe 23:498-510 e495. 10.1016/j.chom.2018.03.003

Gao LL, and Xue HW. 2012. Global analysis of expression profiles of rice receptor-like kinase genes. Mol Plant 5:143-153. 10.1093/mp/ssr062

Girija AM, Kinathi BK, Madhavi MB, Ramesh P, Vungarala S, Patel HK, and Sonti RV. 2017. Rice Leaf Transcriptional Profiling Suggests a Functional Interplay Between Xanthomonas oryzae pv. oryzae Lipopolysaccharide and Extracellular Polysaccharide in Modulation of Defense Responses During Infection. Molecular Plant-Microbe Interactions 30:16-27. 10.1094/MPMI-08-16-0157-R

Gomez-Gomez L, and Boller T. 2000. FLS2: an LRR receptor-like kinase involved in the perception of the bacterial elicitor flagellin in Arabidopsis. Molecular Cell 5:1003-1011.

Grison MS, Kirk P, Brault ML, Wu XN, Schulze WX, Benitez-Alfonso Y, Immel F, and Bayer EM. 2019. Plasma Membrane-Associated Receptor-like Kinases Relocalize to Plasmodesmata in Response to Osmotic Stress. Plant Physiology 181:142-160. 10.1104/pp.19.00473

Hord CL, Chen C, Deyoung BJ, Clark SE, and Ma H. 2006. The BAM1/BAM2 receptor-like kinases are important regulators of Arabidopsis early anther development. Plant Cell 18:1667-1680. 10.1105/tpc. 105.036871

Hussain S, Yin H, Peng S, Khan FA, Khan F, Sameeullah M, Hussain HA, Huang J, Cui K, and Nie L. 2016. Comparative Transcriptional Profiling of Primed and Non-primed Rice Seedlings under Submergence Stress. Frontiers of Plant Science 7:1125. 10.3389/fpls.2016.01125

Jinn TL, Stone JM, and Walker JC. 2000. HAESA, an Arabidopsis leucine-rich repeat receptor kinase, controls floral organ abscission. Genes \& Development 14:108-117.

Kachroo A, Schopfer CR, Nasrallah ME, and Nasrallah JB. 2001. Allele-specific receptor-ligand interactions in Brassica self-incompatibility. Science 293:1824-1826. 10.1126/science. 1062509

Kim HS, Jung MS, Lee SM, Kim KE, Byun H, Choi MS, Park HC, Cho MJ, and Chung WS. 2009. An S-locus receptor-like kinase plays a role as a negative regulator in plant defense responses. Biochemical and Biophysical Research Communications 381:424428. 10.1016/j.bbrc.2009.02.050

Lehti-Shiu MD, and Shiu SH. 2012. Diversity, classification and function of the plant protein kinase superfamily. Philosophical Transactions of the Royal Society of London B Biological Sciences 367:2619-2639. 10.1098/rstb.2012.0003

Lehti-Shiu MD, Zou C, Hanada K, and Shiu SH. 2009. Evolutionary history and stress regulation of plant receptor-like kinase/pelle genes. Plant Physiology 150:12-26. 10.1104/pp.108.134353

Letunic I, and Bork P. 2016. Interactive tree of life (iTOL) v3: an online tool for the display and annotation of phylogenetic and other trees. Nucleic Acids Res 44:W242-245. 10.1093/nar/gkw290

Li Y, Tang D, Li L, Zhao X, Lin J, and Liu X. 2018. Plant Stature Related receptor-like Kinanse2 (PSRK2) acts as a factor that determines stem elongation toward gibberellins response in rice. Bioscience Biotechnology and Biochemistry 82:1931-1941. 10.1080/09168451.2018.1501266

Lima JM, Nath M, Dokku P, Raman KV, Kulkarni KP, Vishwakarma C, Sahoo SP, Mohapatra UB, Mithra SV, Chinnusamy V, Robin S, Sarla N, Seshashayee M, Singh K, Singh AK, Singh NK, Sharma RP, and Mohapatra T. 2015. Physiological, anatomical and transcriptional alterations in a rice mutant leading to enhanced water stress tolerance. AoB Plants 7. 10.1093/aobpla/plv023

Lin CC, Chao YT, Chen WC, Ho HY, Chou MY, Li YR, Wu YL, Yang HA, Hsieh H, Lin CS, Wu FH, Chou SJ, Jen HC, Huang YH, Irene D, Wu WJ, Wu JL, Gibbs DJ, Ho MC, and Shih

Peer) reviewing PDF | (2020:07:51145:3:0:NEW 25 Jan 2021) 
804

805

806

807

808

809

810

811

812

813

814

815

816

817

818

819

820

821

822

823

824

825

826

827

828

829

830

831

832

833

834

835

836

837

838

839

840

841

842

843

844

845

846

847

848

849

850

851

852

853

MC. 2019. Regulatory cascade involving transcriptional and $\mathrm{N}$-end rule pathways in rice under submergence. Proceedings of the National Academy of Sciences of the United States of America 116:3300-3309. 10.1073/pnas.1818507116

Liu F, Xu W, Wei Q, Zhang Z, Xing Z, Tan L, Di C, Yao D, Wang C, Tan Y, Yan H, Ling Y, Sun C, Xue Y, and Su Z. 2010. Gene expression profiles deciphering rice phenotypic variation between Nipponbare (Japonica) and 93-11 (Indica) during oxidative stress. PLOS ONE 5:e8632. 10.1371/journal.pone.0008632

Ma R, Han Z, Hu Z, Lin G, Gong X, Zhang H, Nasrallah JB, and Chai J. 2016. Structural basis for specific self-incompatibility response in Brassica. Cell Research 26:1320-1329. 10.1038/cr.2016.129

Magbanua ZV, Arick M, 2nd, Buza T, Hsu CY, Showmaker KC, Chouvarine P, Deng P, Peterson DG, and Lu S. 2014. Transcriptomic dissection of the rice-Burkholderia glumae interaction. BMC Genomics 15:755. 10.1186/1471-2164-15-755

Myburg AA, Grattapaglia D, Tuskan GA, Hellsten U, Hayes RD, Grimwood J, Jenkins J, Lindquist E, Tice H, Bauer D, Goodstein DM, Dubchak I, Poliakov A, Mizrachi E, Kullan AR, Hussey SG, Pinard D, van der Merwe K, Singh P, van Jaarsveld I, Silva-Junior OB, Togawa RC, Pappas MR, Faria DA, Sansaloni CP, Petroli CD, Yang X, Ranjan P, Tschaplinski TJ, Ye CY, Li T, Sterck L, Vanneste K, Murat F, Soler M, Clemente HS, Saidi N, Cassan-Wang H, Dunand C, Hefer CA, Bornberg-Bauer E, Kersting AR, Vining K, Amarasinghe V, Ranik M, Naithani S, Elser J, Boyd AE, Liston A, Spatafora JW, Dharmwardhana P, Raja R, Sullivan C, Romanel E, Alves-Ferreira M, Kulheim C, Foley W, Carocha V, Paiva J, Kudrna D, Brommonschenkel SH, Pasquali G, Byrne M, Rigault P, Tibbits J, Spokevicius A, Jones RC, Steane DA, Vaillancourt RE, Potts BM, Joubert F, Barry K, Pappas GJ, Strauss SH, Jaiswal P, Grima-Pettenati J, Salse J, Van de Peer Y, Rokhsar DS, and Schmutz J. 2014. The genome of Eucalyptus grandis. Nature 510:356362. 10.1038/nature13308

Naithani S, Chookajorn T, Ripoll DR, and Nasrallah JB. 2007. Structural modules for receptor dimerization in the S-locus receptor kinase extracellular domain. Proceedings of the National Academy of Sciences of the United States of America 104:12211-12216. 10.1073/pnas.0705186104

Naithani S, Dharmawardhana P, and Nasrallah JB. 2013. SCR. In: Kastin AJ, ed. Handbook of Biologically Active Peptides. 2nd ed: Academic Press, 58-66.

Naithani S, Gupta P, Preece J, D'Eustachio P, Elser JL, Garg P, Dikeman DA, Kiff J, Cook J, Olson A, Wei S, Tello-Ruiz MK, Mundo AF, Munoz-Pomer A, Mohammed S, Cheng T, Bolton E, Papatheodorou I, Stein L, Ware D, and Jaiswal P. 2020. Plant Reactome: a knowledgebase and resource for comparative pathway analysis. Nucleic Acids Res 48:D1093-D1103. 10.1093/nar/gkz996

Naithani S, Gupta P, Preece J, Garg P, Fraser V, Padgitt-Cobb LK, Martin M, Vining K, and Jaiswal P. 2019. Involving community in genes and pathway curation. Database (Oxford) 2019. 10.1093/database/bay146

Naithani S, Nonogaki H, and Jaiswal P. 2017. Exploring crossroads between seed development and stress-response. In: Pandey GK, ed. Mechanism of Plant Hormone Signaling under Stress. New Jersey, USA: John Wiley \& Sons, Inc. , 415-454.

Nandakumar R, Shahjahan AKM, Yuan XL, Dickstein ER, Groth DE, Clark CA, Cartwright RD, and Rush MC. 2009. Burkholderia glumae and B. gladioli Cause Bacterial Panicle Blight in Rice in the Southern United States. Plant Disease 93:896-905. 10.1094/PDIS-93-90896

Narsai R, Wang C, Chen J, Wu J, Shou H, and Whelan J. 2013. Antagonistic, overlapping and distinct responses to biotic stress in rice (Oryza sativa) and interactions with abiotic stress. BMC Genomics 14:93. 10.1186/1471-2164-14-93 
854

855

856

857

858

859

860

861

862

863

864

865

866

867

868

869

870

871

872

873

874

875

876

877

878

879

880

881

882

883

884

885

886

887

888

889

890

891

892

893

894

895

896

897

898

899

900

901

902

903

Ouyang SQ, Liu YF, Liu P, Lei G, He SJ, Ma B, Zhang WK, Zhang JS, and Chen SY. 2010. Receptor-like kinase OsSIK1 improves drought and salt stress tolerance in rice (Oryza sativa) plants. Plant Journal 62:316-329. 10.1111/j.1365-313X.2010.04146.x

Pan J, Li Z, Wang Q, Yang L, Yao F, and Liu W. 2020. An S-domain receptor-like kinase, OsESG1, regulates early crown root development and drought resistance in rice. Plant Science 290:110318. 10.1016/j.plantsci.2019.110318

Papatheodorou I, Moreno P, Manning J, Fuentes AM, George N, Fexova S, Fonseca NA, Fullgrabe A, Green M, Huang N, Huerta L, lqbal H, Jianu M, Mohammed S, Zhao L, Jarnuczak AF, Jupp S, Marioni J, Meyer K, Petryszak R, Prada Medina CA, TalaveraLopez C, Teichmann S, Vizcaino JA, and Brazma A. 2020. Expression Atlas update: from tissues to single cells. Nucleic Acids Res 48:D77-D83. 10.1093/nar/gkz947

Pruitt RN, Schwessinger B, Joe A, Thomas N, Liu F, Albert M, Robinson MR, Chan LJ, Luu DD, Chen H, Bahar O, Daudi A, De Vleesschauwer D, Caddell D, Zhang W, Zhao X, Li X, Heazlewood JL, Ruan D, Majumder D, Chern M, Kalbacher H, Midha S, Patil PB, Sonti RV, Petzold CJ, Liu CC, Brodbelt JS, Felix G, and Ronald PC. 2015. The rice immune receptor XA21 recognizes a tyrosine-sulfated protein from a Gram-negative bacterium. Sci Adv 1:e1500245. 10.1126/sciadv.1500245

Pu CX, Han YF, Zhu S, Song FY, Zhao Y, Wang CY, Zhang YC, Yang Q, Wang J, Bu SL, Sun LJ, Zhang SW, Zhang SQ, Sun DY, and Sun Y. 2017. The Rice Receptor-Like Kinases DWARF AND RUNTISH SPIKELET1 and 2 Repress Cell Death and Affect Sugar Utilization during Reproductive Development. Plant Cell 29:70-89. 10.1105/tpc.16.00218

Sakai H, Mizuno H, Kawahara Y, Wakimoto H, Ikawa H, Kawahigashi H, Kanamori H, Matsumoto T, Itoh T, and Gaut BS. 2011. Retrogenes in rice (Oryza sativa L. ssp. japonica) exhibit correlated expression with their source genes. Genome Biol Evol 3:1357-1368. 10.1093/gbe/evr111

Shiu SH, and Bleecker AB. 2001a. Plant receptor-like kinase gene family: diversity, function, and signaling. Sci STKE 2001:re22. 10.1126/stke.2001.113.re22

Shiu SH, and Bleecker AB. 2001b. Receptor-like kinases from Arabidopsis form a monophyletic gene family related to animal receptor kinases. Proceedings of the National Academy of Sciences of the United States of America 98:10763-10768. 10.1073/pnas.181141598

Shiu SH, and Bleecker AB. 2003. Expansion of the receptor-like kinase/Pelle gene family and receptor-like proteins in Arabidopsis. Plant Physiology 132:530-543. 10.1104/pp.103.021964

Shiu SH, Karlowski WM, Pan R, Tzeng YH, Mayer KF, and Li WH. 2004. Comparative analysis of the receptor-like kinase family in Arabidopsis and rice. Plant Cell 16:1220-1234. $10.1105 /$ tpc. 020834

Shpak ED, Lakeman MB, and Torii KU. 2003. Dominant-negative receptor uncovers redundancy in the Arabidopsis ERECTA Leucine-rich repeat receptor-like kinase signaling pathway that regulates organ shape. Plant Cell 15:1095-1110. $10.1105 /$ tpc. 010413

Sievers F, Wilm A, Dineen D, Gibson TJ, Karplus K, Li W, Lopez R, McWilliam H, Remmert M, Soding J, Thompson JD, and Higgins DG. 2011. Fast, scalable generation of highquality protein multiple sequence alignments using Clustal Omega. Molecular Systems Biology 7:539. 10.1038/msb.2011.75

Stein JC, Howlett B, Boyes DC, Nasrallah ME, and Nasrallah JB. 1991. Molecular cloning of a putative receptor protein kinase gene encoded at the self-incompatibility locus of Brassica oleracea. Proceedings of the National Academy of Sciences of the United States of America 88:8816-8820.

Takayama S, and Isogai A. 2003. Molecular mechanism of self-recognition in Brassica selfincompatibility. Journal of Experimental Botany 54:149-156.

Peer) reviewing PDF | (2020:07:51145:3:0:NEW 25 Jan 2021) 
904

905

906

907

908

909

910

911

912

913

914

915

916

917

918

919

920

921

922

923

924

925

926

927

928

929

930

931

932

933

934

935

936

937

938

939

940

941

942

943

944

945

946
Tantikanjana T, Rizvi N, Nasrallah ME, and Nasrallah JB. 2009. A dual role for the S-locus receptor kinase in self-incompatibility and pistil development revealed by an Arabidopsis rdr6 mutation. Plant Cell 21:2642-2654. 10.1105/tpc.109.067801

Tello-Ruiz MK, Naithani S, Stein JC, Gupta P, Campbell M, Olson A, Wei S, Preece J, Geniza MJ, Jiao Y, Lee YK, Wang B, Mulvaney J, Chougule K, Elser J, Al-Bader N, Kumari S, Thomason J, Kumar V, Bolser DM, Naamati G, Tapanari E, Fonseca N, Huerta L, Iqbal H, Keays M, Munoz-Pomer Fuentes A, Tang A, Fabregat A, D'Eustachio P, Weiser J, Stein LD, Petryszak R, Papatheodorou I, Kersey PJ, Lockhart P, Taylor C, Jaiswal P, and Ware D. 2018. Gramene 2018: unifying comparative genomics and pathway resources for plant research. Nucleic Acids Res 46:D1181-D1189. 10.1093/nar/gkx1111

Vining KJ, Romanel E, Jones RC, Klocko A, Alves-Ferreira M, Hefer CA, Amarasinghe V, Dharmawardhana P, Naithani S, Ranik M, Wesley-Smith J, Solomon L, Jaiswal P, Myburg AA, and Strauss SH. 2015. The floral transcriptome of Eucalyptus grandis. New Phytologist 206:1406-1422. 10.1111/nph.13077

Wang J, Qu B, Dou S, Li L, Yin D, Pang Z, Zhou Z, Tian M, Liu G, Xie Q, Tang D, Chen X, and Zhu L. 2015. The E3 ligase OsPUB15 interacts with the receptor-like kinase PID2 and regulates plant cell death and innate immunity. BMC Plant Biology 15:49. $10.1186 / \mathrm{s} 12870-015-0442-4$

Wolf S. 2017. Plant cell wall signalling and receptor-like kinases. Biochemical Journal 474:471492. 10.1042/BCJ20160238

Wu YS, and Yang CY. 2020. Comprehensive Transcriptomic Analysis of Auxin Responses in Submerged Rice Coleoptile Growth. Int J Mol Sci 21. 10.3390/ijms21041292

Yu TY, Shi DQ, Jia PF, Tang J, Li HJ, Liu J, and Yang WC. 2016. The Arabidopsis Receptor Kinase ZAR1 Is Required for Zygote Asymmetric Division and Its Daughter Cell Fate. PLoS Genetics 12:e1005933. 10.1371/journal.pgen.1005933

Zhai R, Feng Y, Wang H, Zhan X, Shen X, Wu W, Zhang Y, Chen D, Dai G, Yang Z, Cao L, and Cheng S. 2013. Transcriptome analysis of rice root heterosis by RNA-Seq. BMC Genomics 14:19. 10.1186/1471-2164-14-19

Zhang T, Zhao X, Wang W, Pan Y, Huang L, Liu X, Zong Y, Zhu L, Yang D, and Fu B. 2012. Comparative transcriptome profiling of chilling stress responsiveness in two contrasting rice genotypes. PLoS ONE 7:e43274. 10.1371/journal.pone.0043274

Zhang YC, Liao JY, Li ZY, Yu Y, Zhang JP, Li QF, Qu LH, Shu WS, and Chen YQ. 2014. Genome-wide screening and functional analysis identify a large number of long noncoding RNAs involved in the sexual reproduction of rice. Genome Biology 15:512. 10.1186/s13059-014-0512-1

Zhao W, Yang P, Kang L, and Cui F. 2016. Different pathogenicities of Rice stripe virus from the insect vector and from viruliferous plants. New Phytologist 210:196-207. 10.1111/nph.13747

Zou X, Qin Z, Zhang C, Liu B, Liu J, Zhang C, Lin C, Li H, and Zhao T. 2015. Over-expression of an S-domain receptor-like kinase extracellular domain improves panicle architecture and grain yield in rice. Journal of Experimental Botany 66:7197-7209. 10.1093/jxb/erv417 


\section{Figure 1}

Variations in the domain structure of SDRLK family proteins.

(A) Canonical domain structure of the SDRLK gene family's prototypic member, the S-locus receptor kinase SRK based on previously published data (Naithani et al. 2007; Ma et al. 2016). (B) Predicted domain structure for SRK2-b allele of Brassica oleracea and (C) domain structure of the 144 rice SDRLK family proteins based on information available in the UniProt. Bulb-Type Lectin-like domain (LLD), EGF-like, PAN/HGF, Transmembrane (TM), and Ser/Thr Kinase domains. The "S-locus glycoprotein" (SLG) sequence motif is shared among various SRK alleles and SRK family genes. 
(A)

SRK

\begin{tabular}{|l|c|}
\hline SP & LLD1 \\
\hline
\end{tabular}

LLD2

EGF-like

PAN/HGF

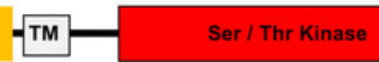

Deduced

(B)

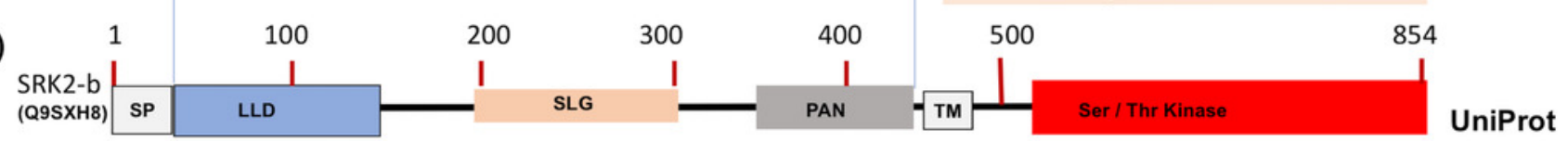

(C)

Vaiations in the domain structure of rice (O. sativa) SD-RLK family proteins

\begin{tabular}{|c|c|c|c|c|c|c|c|c|c|c|c|}
\hline Rice SD-RLKs (total 144) & Conserved Domains (UniProt) & \#Domains & \#Proteins & SP & LLD & SLG & EGF & PAN-apple & $\mathrm{TM}$ & Kinase & Gnk \\
\hline \multirow{8}{*}{ Receptor-like Kinases (67) } & $\mathrm{SP}+\mathrm{LLD}+\mathrm{SLG}+\mathrm{EGF}+\mathrm{PAN}$-apple + TM + Kinase & 7 & 8 & Yes & Yes & Yes & Yes & Yes & Yes & Yes & \\
\hline & LLD + SLG + EGF + PAN-apple + TM + Kinase & 6 & 2 & No & Yes & Yes & Yes & Yes & Yes & Yes & \\
\hline & SP + LLD + SLG + PAN-apple + TM + Kinase & 6 & 38 & Yes & Yes & Yes & No & Yes & Yes & Yes & \\
\hline & SP + LLD + EGF + PAN-apple + TM + Kinase & 6 & 4 & Yes & Yes & No & Yes & Yes & Yes & Yes & \\
\hline & SP + LLD + PAN-apple + TM + Kinase & 5 & 2 & Yes & Yes & No & No & Yes & Yes & Yes & \\
\hline & LLD + SLG + PAN-apple + TM + Kinase & 5 & 10 & No & Yes & Yes & No & Yes & Yes & Yes & \\
\hline & $\mathrm{SP}+\mathrm{LLD}+\mathrm{SLG}+\mathrm{EGF}+\mathrm{TM}+$ Kinase & 6 & 2 & Yes & Yes & Yes & Yes & No & Yes & Yes & \\
\hline & LLD+SLG+EGF+TM+Kinase & 5 & 1 & no & yes & yes & yes & No & yes & yes & \\
\hline \multirow{6}{*}{ Truncated SD-RLKs (15) } & $S P+L D+T M+K i n a s e$ & 4 & 7 & Y & & & & & & & \\
\hline & $L L D+S L G+T M+$ Kinase & 4 & 1 & No & Yes & Yes & No & No & Yes & Yes & \\
\hline & LLD + PAN-apple + TM + Kinase & 4 & 1 & No & Yes & No & No & Yes & Yes & Yes & \\
\hline & PAN-apple + TM + Kinase & 3 & 1 & No & No & No & No & Yes & Yes & Yes & \\
\hline & $\mathrm{SLG}+\mathrm{TM}+$ Kinase & 3 & 2 & No & No & Yes & No & No & Yes & Yes & \\
\hline & SLG + PAN-apple + TM + Kinase & 4 & 3 & No & No & Yes & No & Yes & Yes & Yes & \\
\hline \multirow{10}{*}{ Receptor-like proteins (12) } & $S P+L L D+S L G$ & 3 & 2 & Yes & Yes & Yes & No & No & No & No & \\
\hline & SP + LLD + SLG + PAN-apple & 5 & 2 & Yes & Yes & Yes & No & Yes & no & No & \\
\hline & $S P+L L D+S L G+E G F+T M$ & 6 & 1 & Yes & Yes & Yes & Yes & Yes & No & No & \\
\hline & $L L D+S L G+E G F+T M$ & 4 & 1 & No & Yes & Yes & Yes & No & Yes & No & \\
\hline & $\mathrm{SP}+\mathrm{LLD}+\mathrm{SLG}+\mathrm{PAN}$-apple+TM & 5 & 1 & Yes & Yes & Yes & Yes & Yes & No & No & \\
\hline & LLD + EGF + PAN-apple + TM & 4 & 1 & No & Yes & No & Yes & Yes & Yes & No & \\
\hline & SLG + PAN-apple + TM & 3 & 1 & No & No & Yes & No & Yes & Yes & No & \\
\hline & $S P+L L D+T M$ & 3 & 1 & Yes & Yes & No & No & No & Yes & No & \\
\hline & LLD & 1 & 1 & No & Yes & No & No & No & No & No & \\
\hline & $\mathrm{LLD}+\mathrm{SLG}+\mathrm{PAN}$-apple + TM & 4 & 1 & No & Yes & Yes & No & Yes & Yes & No & \\
\hline \multirow{4}{*}{ SD-Kinases (14) } & SP + LLD + SLG + EGF + PAN-apple + Kinase & 6 & 3 & yes & yes & yes & yes & yes & no & yes & \\
\hline & SP + LLD + SLG + PAN-apple + Kinase & 5 & 9 & yes & yes & yes & no & yes & no & yes & \\
\hline & LLD + SLG + Kinase & 3 & 1 & no & yes & yes & no & no & no & yes & \\
\hline & $L L D+S L G$ + PAN-apple + Kinase & 4 & 1 & no & yes & yes & no & yes & no & yes & \\
\hline \multirow{2}{*}{ Other Kinases (26) } & $\mathrm{TM}+$ Kinase & 2 & 12 & No & No & No & No & No & Yes & Yes & \\
\hline & Kinase & 1 & 14 & No & No & No & No & No & No & Yes & \\
\hline \multirow{3}{*}{ Kinases + GnK2 domain (10) } & Gnk + Kinase & 2 & 1 & No & No & No & No & No & No & Yes & New \\
\hline & Gnk + TM + Kinase & 3 & 1 & No & No & No & No & No & Yes & Yes & New \\
\hline & $\mathrm{SP}+\mathrm{G} n \mathrm{k}+\mathrm{TM}+$ Kinase & 4 & 8 & Yes & No & No & No & No & Yes & Yes & New \\
\hline
\end{tabular}


Figure 2

The rice SDRLK family genes differ in their tissue-specific expression..

The heat map of the expression data was generated based on the hierarchical clustering of genes showing similar expression patterns. Datasets: E-MTAB-2039, E-MTAB-2037, EMTAB-4123, and E-GEOD-50777 from EMBL-EBI Gene Expression Atlas. 


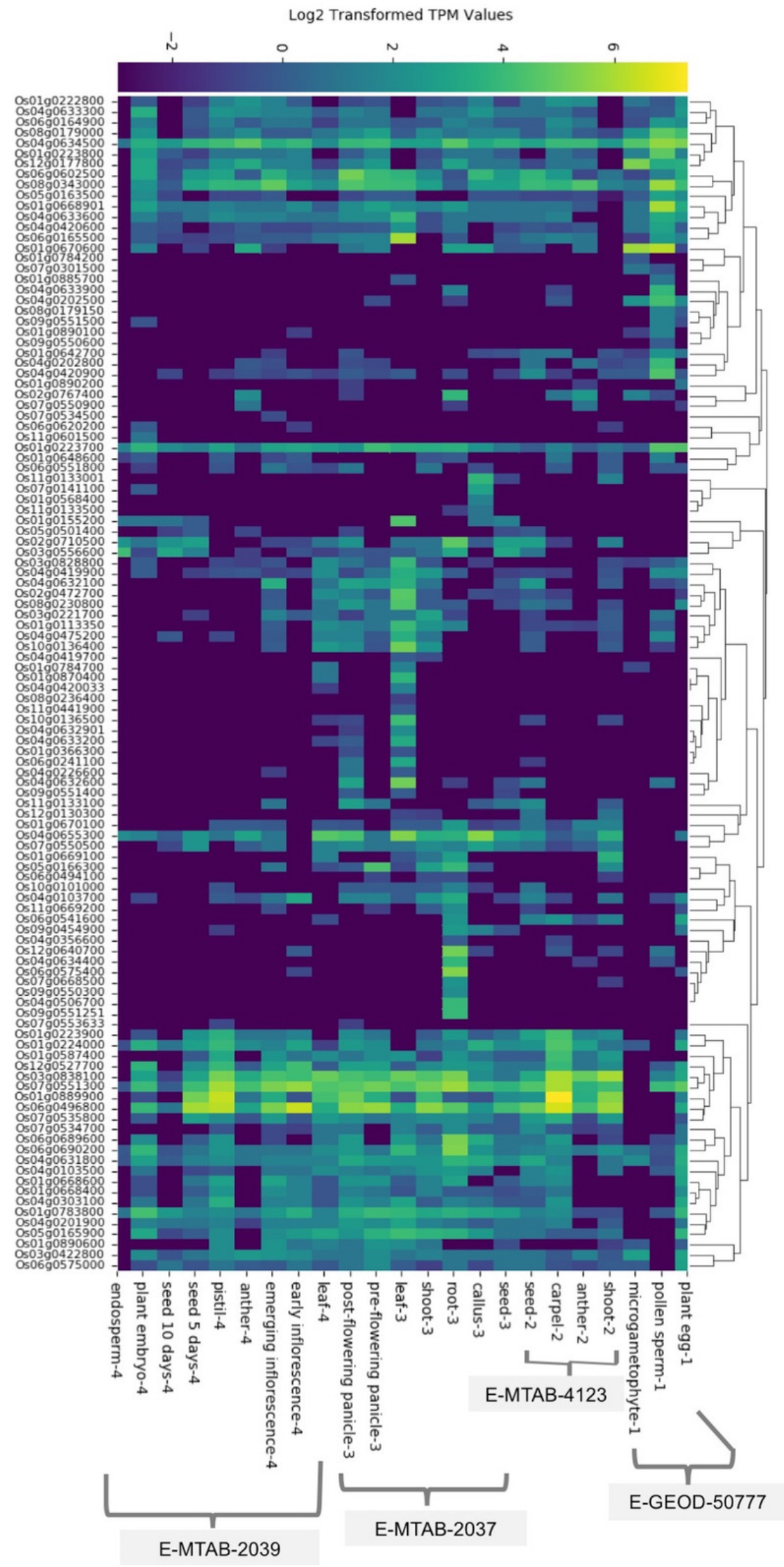




\section{Figure 3}

SDRLK genes are regulated in response to bacterial pathogen $X$. oryzae.

(A) Differential gene expression in rice leaves infected with three $X$. oryzae pv. oryzae (Xoo) strains, BXO43 (wild-type), BX01002 (EPS), and BX01003 (LPS',EPS). (B) Differential expression of genes in two susceptible rice varieties IR24 and Nipponbare in response to various $X$. oryzae strains. (C) Changes in gene expression in response to virulent $X o 0$ strain PX071 in a susceptible IR24 and a resistant IRRBB21 genotypes after 24 and 96 hours postinfection. 
(A)

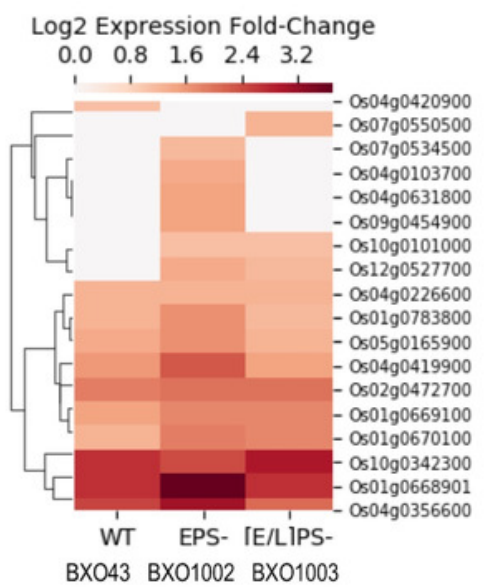

(C)
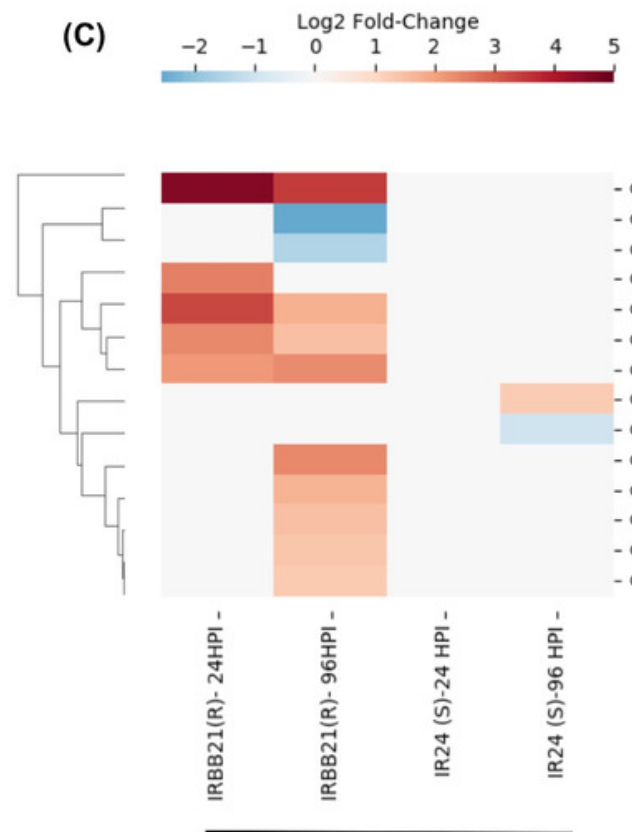

Xoo strain PX071
(B)

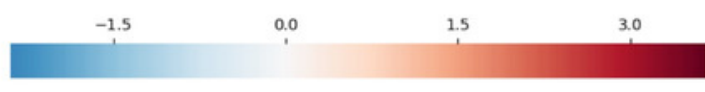

Os 1090136400 -

Os 10 g0 0136500 -

Os01g0223700 -

Os01g0784700 -

Os0190890600 -

Os04g0420033 -

Os03g0221700 -

OS04g0419900 -

Os0690165500 -

Os07g0534700 -

Os0890179000

Os04g0632600 -

Os0190670600 -

Os0390422800

Os03g0556600 -

Os05g0166300 -

Os06g0494100 -

Os01g0668600

Os0190783800 -

Os02g0472700

Os06g0496800 -

Os07g0550900

Os04g0419700 -

Os12g0527700 -

Os04g0226600 -

Os07g0534500 -

OS0490420900

Os06g0690200 -

Os01g0668901 -

Os04g0103500 -

Os04g0103700 -

Os04g0356600 -

Os04g0631800 -

Os04g0633300

Os05g0165900 -

Os10g0342300 -

Os0190669100 -

Os09g0454900

Os06g0602500

Os0190670100

OS02g0710500
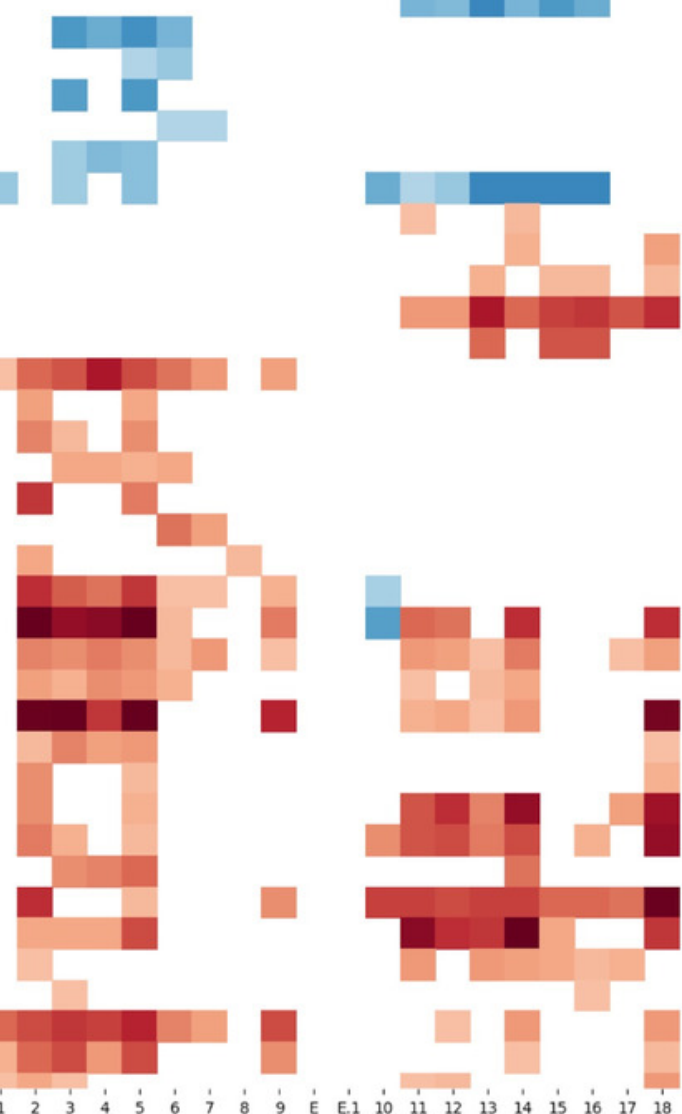

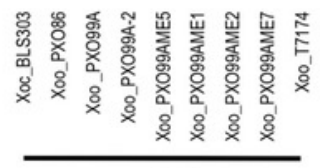

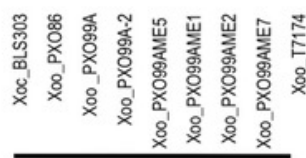

Nipponbare 
Figure 4

Differential expression of SDRLK family genes in response to bacterial pathogen $B$. glumae.

The data is from the seedlings and inflorescence of a moderately resistant (R) CL 161, and a susceptible (S) CL 151 rice genotypes after 48 hours post-inoculation with B. glumae. 


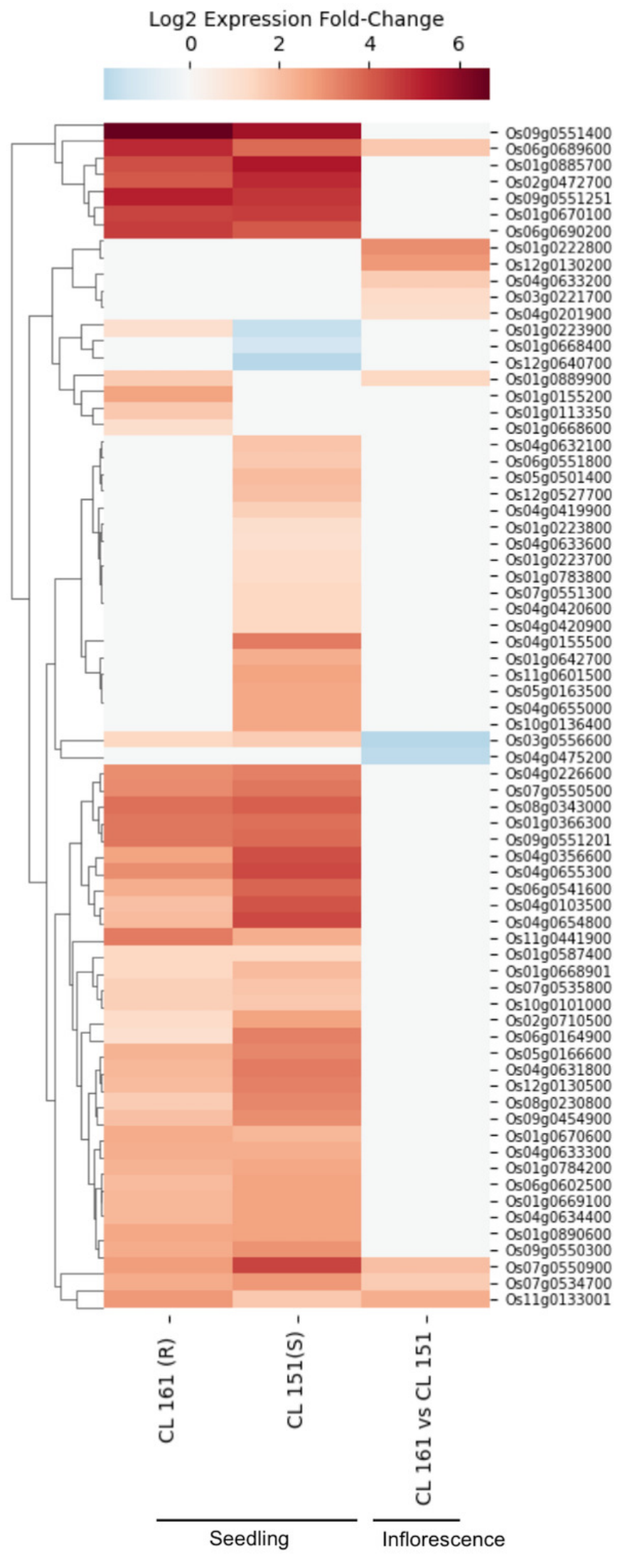

48 hours after $B$. glumae infection 


\section{Figure 5}

Rice SD-RLK family genes are regulated in response to Rice Stripe Virus (RSV) and fungal pathogen $R$. solani.

(A). Change in the expression of SDRLK family genes in rice leaves that were mechanically inoculated with insect-derived RSV and plant-derived RSV in comparison to the rice leaves fed on by the natural viral vector viruliferous $L$. striatellus. (B) Differential expression of the rice SDRLK family genes in response to fungal pathogen $R$. Solani in a susceptible rice variety Cocodrie (CCDR) and a resistant rice variety MCR010277 (MCR) after 3 and 5 days postinfection (DPI). 

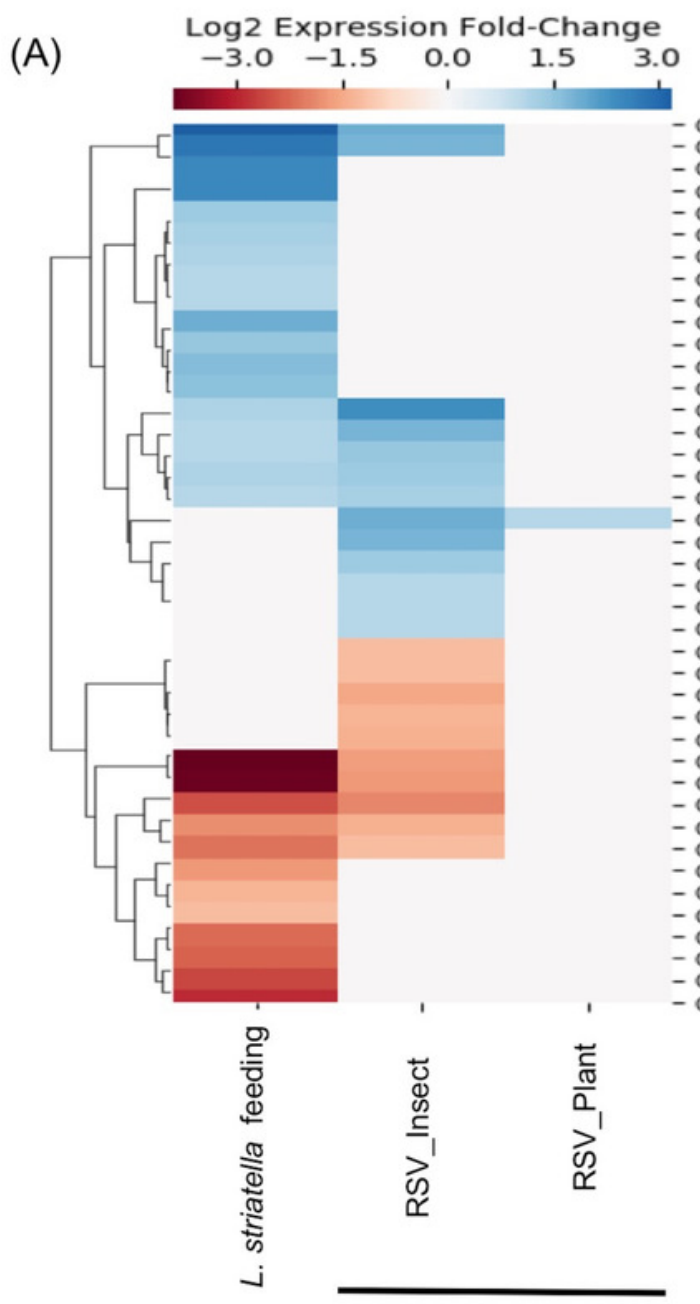

Microinjection

- Os04g0420900 - Os09g0454900 - Os01g0870400 - Os01g0223800 - Os04g0632100 - Os01g0223900 - Os06g0494100 - Os06g0602500 - Os04g0633200 Os10g0136400 - Os10g0136500 - Os12g0640700 - Os07g0550900 - Os02g0710500 - Os04g0103500 Os04g0103500 Os01g0224000 Os07g0551300 - Os01g0670600 - Os0190670100 Os07g0535800 Os04g0419900 Os04g0631800 - Os04g0475200 Os11g0133001 Os0190648600 - Os01g0668600 Os06g0165500 Os12g0130200 - Os12g0130300 - Os11g0133100 - Os01g0890600 - Os12g0527700

Osolgolis3so

- Os01g0669100

- Os01g0889900

- Os04g0654800

Os04g0356600

- Os04g0633300
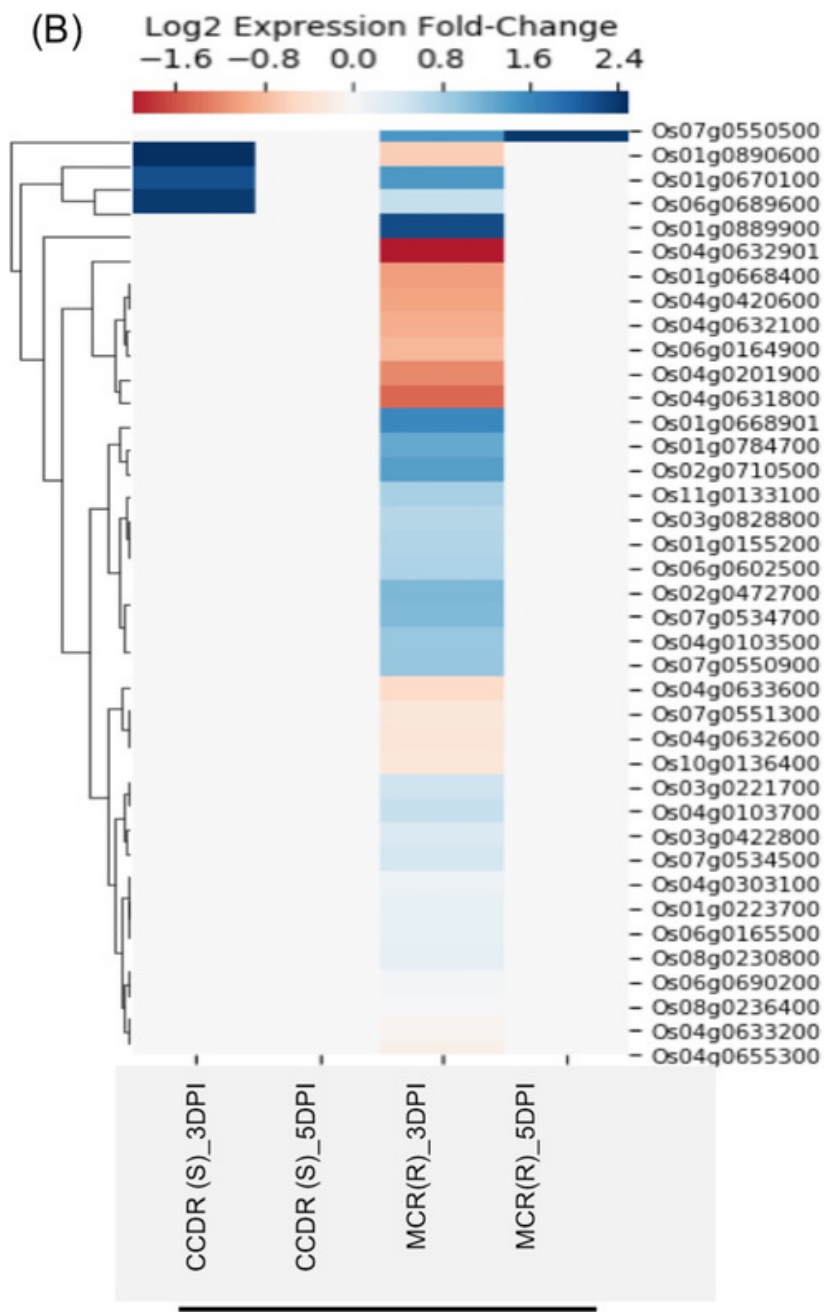

Infected with $R$. solani 
Figure 6

Rice SDRLK family genes show differential expression in response to chilling.

(A) Fold change in the expression of 55 genes in susceptible Thaibonnet and tolerant Volano genotypes treated with chilling stress $\left(10^{\circ} \mathrm{C}\right)$ for 2 and 10 hours (data source: E-MTAB-5941). (B) Differential expression of 59 genes in susceptible IR29 and tolerant LTH genotypes in response to chilling stress at $4^{\circ} \mathrm{C}$ for $2,6,24$, and 48 hours as well as after 24 hours' recovery from 24 hours' stress (data source: E-GEOD-38023). 
(A)

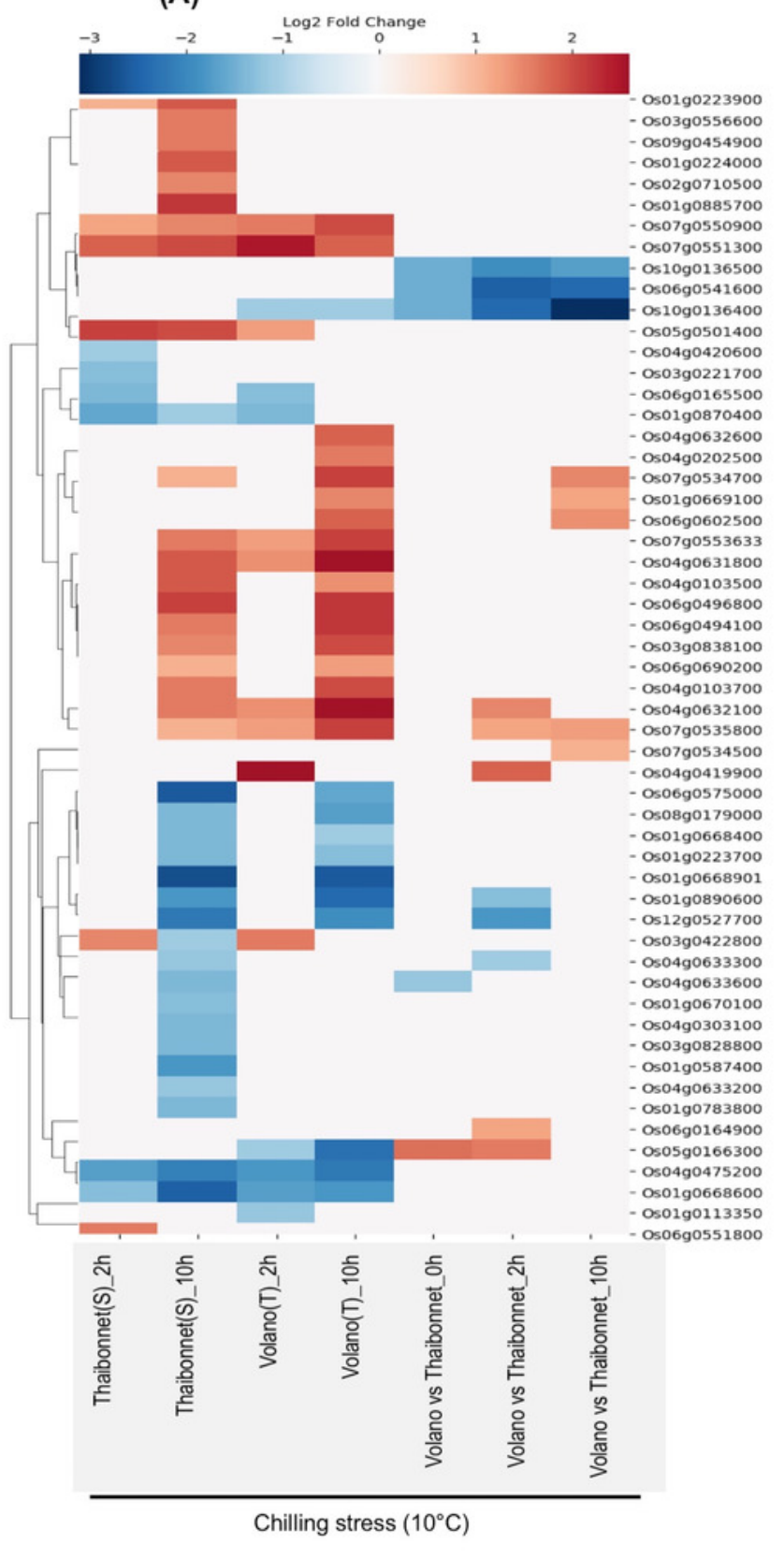

(B)

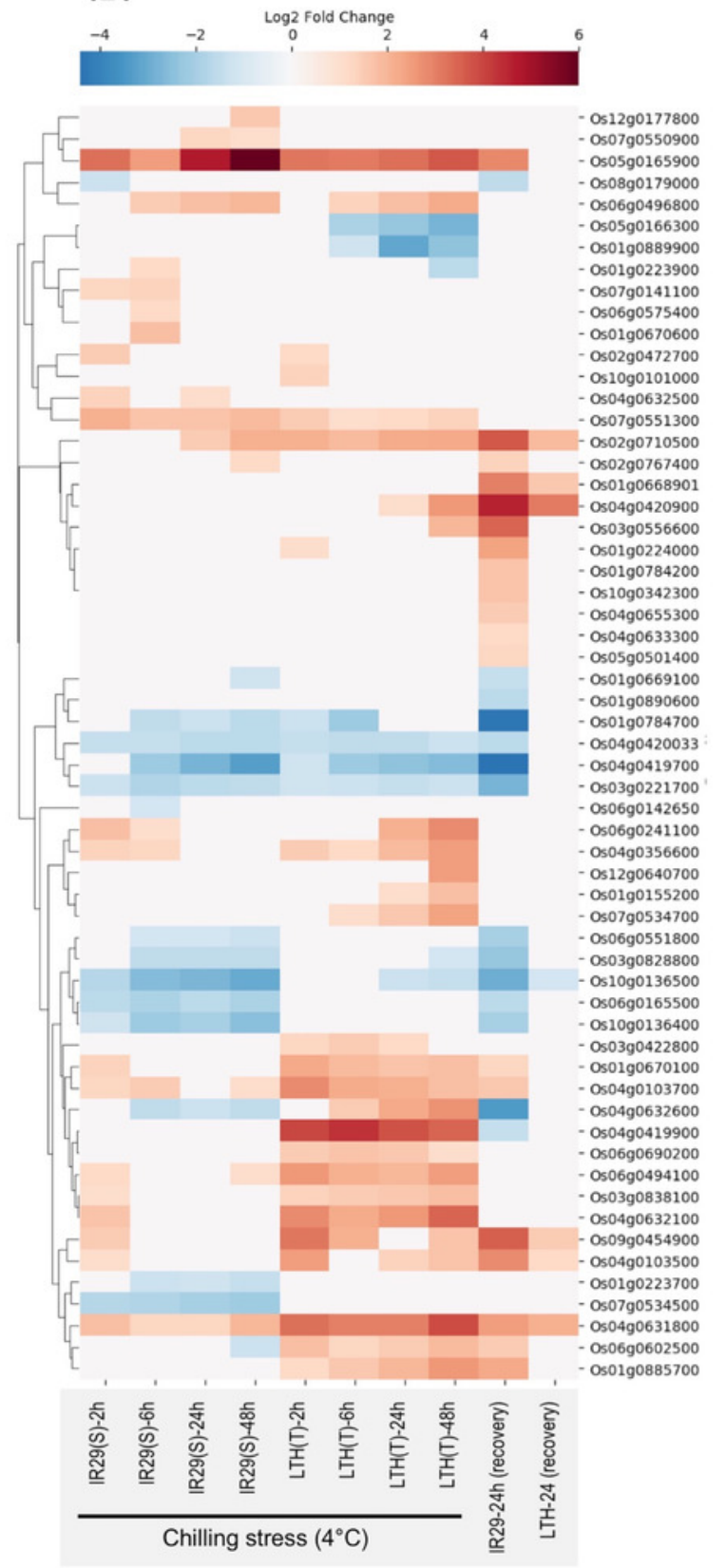




\section{Figure 7}

Rice SDRLK family genes show drought-induced differential gene expression.

Data from four datasets E-GEOD-41647, E-MTAB-4994, E-MTAB-3230, and E-MTAB-6402 was used for comparison across susceptible (S) and tolerant (T) rice genotypes. 


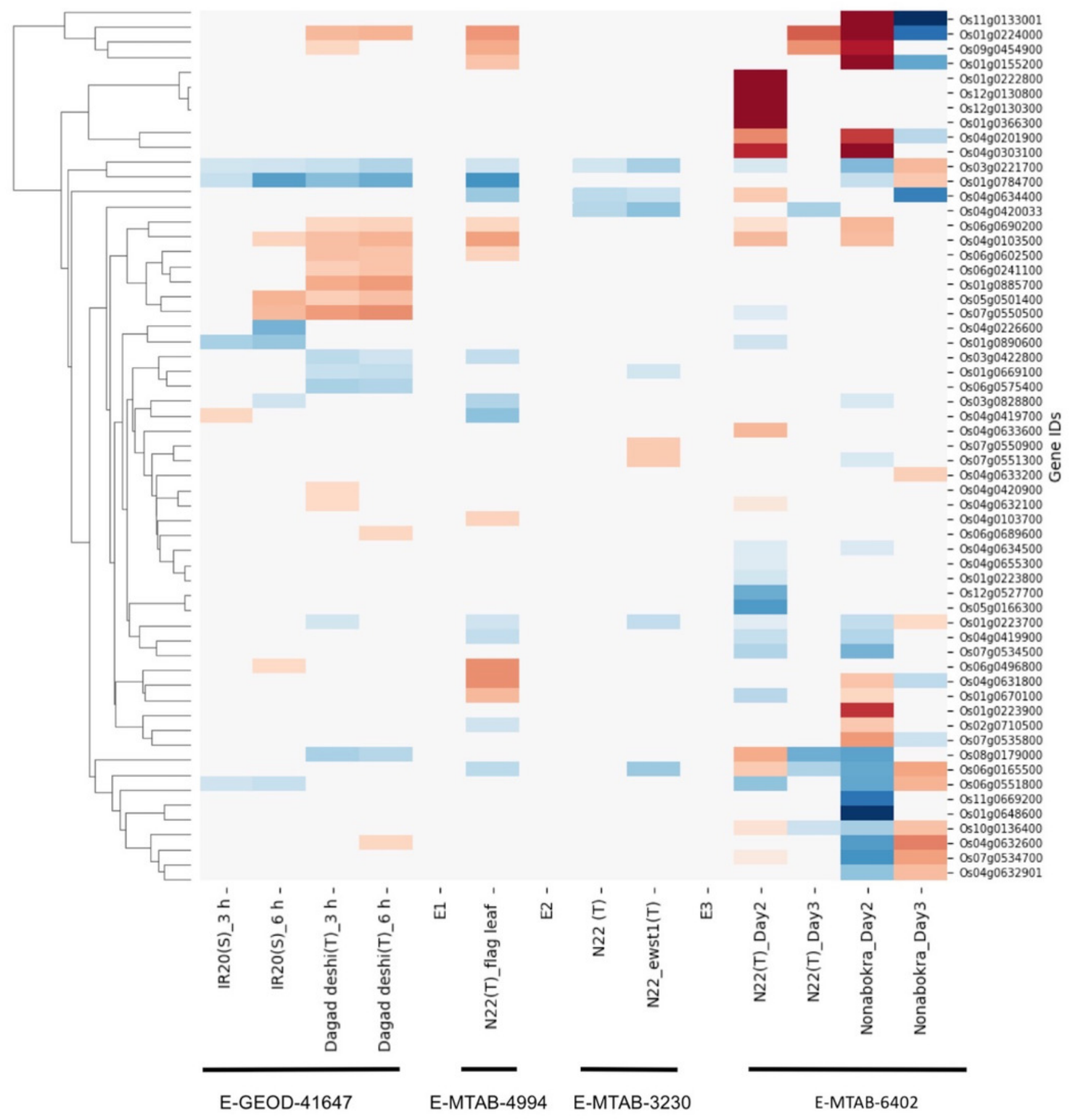


Figure 8

Rice SDRLK family genes are regulated in response to submergence.

Expression data for SDRLK family genes from Wu and Yang (2020) and Hussain et al. (2016) were plotted side by side for comparison between india and japonica rice cultivars under submergence. 


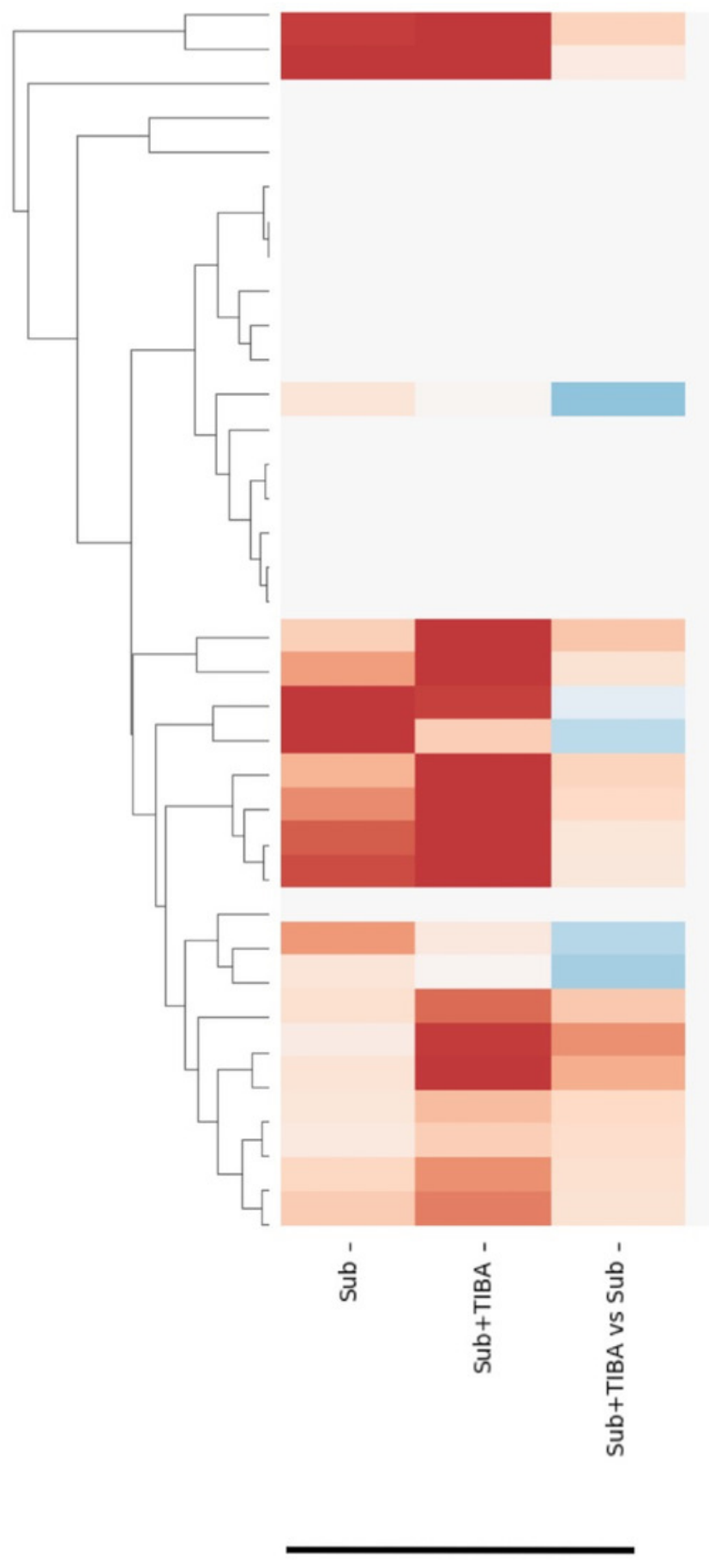

Taikeng 9

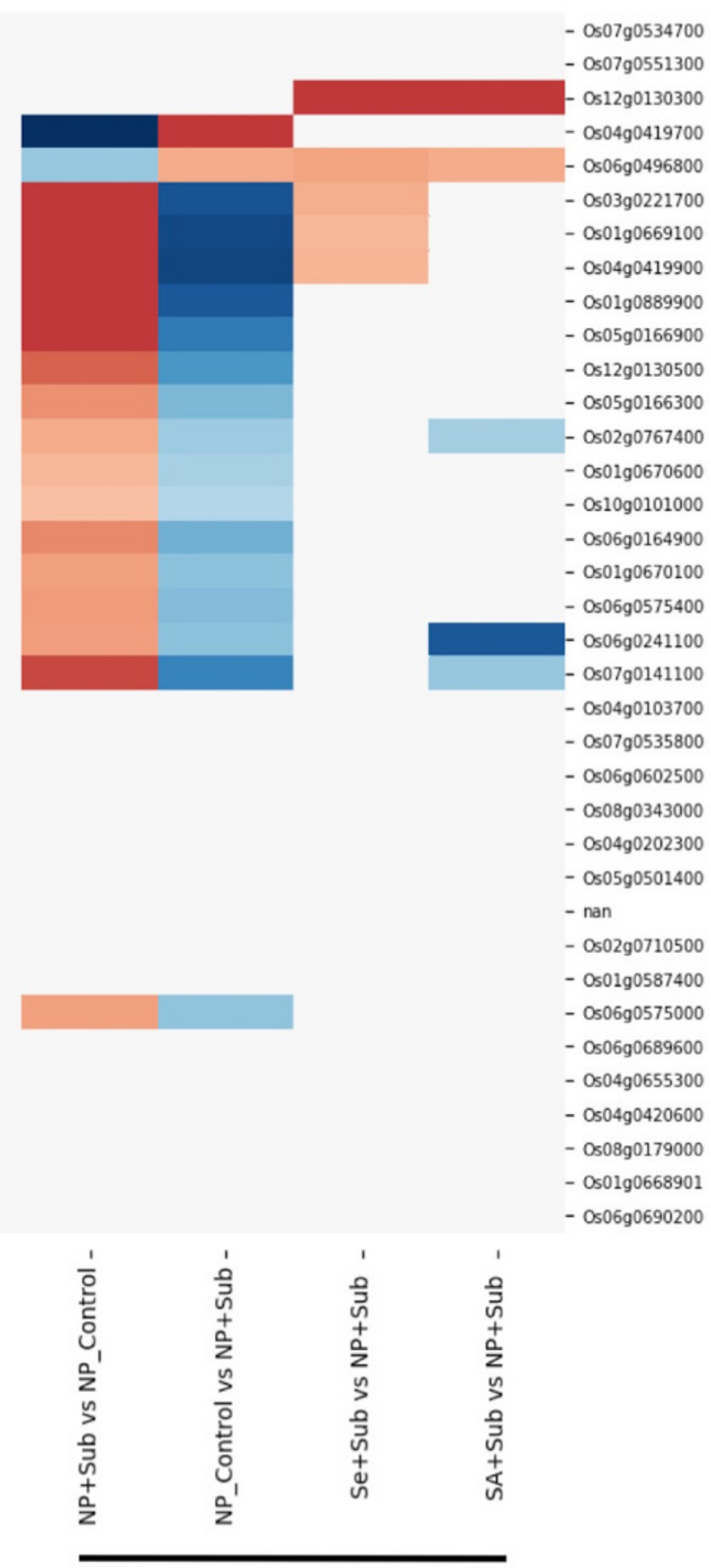

Huanghuazhan 